\title{
Coupled Thermal Field of the Rotor of Liquid Floated Gyroscope
}

\author{
Wang Zhengjun, ${ }^{1}$ Yang Yiyong, ${ }^{1}$ and Yang Jianzhong ${ }^{2,3,4}$ \\ ${ }^{1}$ School of Engineering and Technology, China University of Geosciences (Beijing), Beijing 100083, China \\ ${ }^{2}$ Department of Precision Instruments, Tsinghua University, Beijing 100084, China \\ ${ }^{3}$ Micro-Nano Manufacturing, Collaborative Innovation Center for Devices and Systems, Beijing 100084, China \\ ${ }^{4}$ State Key Laboratory of Precision Measurement Technology and Instruments, Beijing 100084, China \\ Correspondence should be addressed to Yang Yiyong; yangyy@cugb.edu.cn and Yang Jianzhong; yang-jz@tsinghua.edu.cn
}

Received 24 May 2014; Revised 27 August 2014; Accepted 27 August 2014

Academic Editor: Di Zhang

Copyright (C) 2015 Wang Zhengjun et al. This is an open access article distributed under the Creative Commons Attribution License, which permits unrestricted use, distribution, and reproduction in any medium, provided the original work is properly cited.

Inertial navigation devices include star sensor, GPS, and gyroscope. Optical fiber and laser gyroscopes provide high accuracy, and their manufacturing costs are also high. Magnetic suspension rotor gyroscope improves the accuracy and reduces the production cost of the device because of the influence of thermodynamic coupling. Therefore, the precision of the gyroscope is reduced and drift rate is increased. In this study, the rotor of liquid floated gyroscope, particularly the dished rotor gyroscope, was placed under a thermal field, which improved the measurement accuracy of the gyroscope. A dynamic theory of the rotor of liquid floated gyroscope was proposed, and the thermal field of the rotor was simulated. The maximum stress was in $x, 1.4 ; y, 8.43 ;$ min 97.23 ; and $\max 154.34$. This stress occurred at the border of the dished rotor at a high-speed rotation. The secondary flow reached $5549 \mathrm{r} / \mathrm{min}$, and the generated heat increased. Meanwhile, the high-speed rotation of the rotor was volatile, and the dished rotor movement was unstable. Thus, nanomaterials must be added to reduce the thermal coupling fluctuations in the dished rotor and improve the accuracy of the measurement error and drift rate.

\section{Introduction}

MEMS (microelectromechanical systems) gyroscopes, such as micromachined vibrating gyroscope $[1,2]$ and piezoelectric vibrating gyroscope $[3,4]$, are inertial navigation devices that measure angular velocity and altitude angle. They are widely used in numerous fields because of their small volume, light quality, affordability, and high accuracy. Piezoelectric vibrating gyroscope is generally used in electronic products and personal navigation systems $[3,4]$ and its bias stability reaches $0.1^{\circ} / \mathrm{h}$ [5]. However, the mechanical coupling of this kind of vibrating gyroscope causes various problems. Tuning fork gyroscope, whose accuracy reaches the desired theoretical level, was proposed to address such problems [6]. The Shanghai Institute of Microsystem and Information Technology and Shanghai Jiaotong University presented this kind of gyroscope. Beijing University conducted a research on tuning fork gyroscope in 2009 [7, 8]. According to a study conducted by the Georgia Institute of Technology, tuning fork gyroscope exhibits high transition and has a bias drift as low as $0.15^{\circ} / \mathrm{h}$ and ARW of $0.003^{\circ} / \mathrm{h}$, which are the lowest rates among the silicon MEMS gyroscopes. The largest scale factor of a gyroscope is $88 \mathrm{mV} /\left({ }^{\circ} / \mathrm{s}^{-1}\right)$; the bandwidth of the microsystem can be configured between 1 and 10 Hertz [9]. Vibrating ring gyroscope is another kind of gyroscope that has excellent pattern matching, high resolution, low $\mathrm{ZRO}$, and long-term stability. These property parameters are applied in a large number of navigation fields [10-12].

The angular vibration of the MEMS gyroscope is mainly used to measure angle and velocity. The University of Minnesota presented a highly accurate angular vibratory gyroscope [13]. Meanwhile, the University of Michigan presented the integrating rate of a gyroscope based on a 3D gyroscope using high-Q material manufacturing process [14]. The piezoelectric vibrating gyroscope has the following advantages: robustness, wide measurement range, and high resistance to external shocks and shaking. Thus, it can operate in atmospheric environment and vacuum packed environment. In 2009, Hydragog University of Japan proposed a piezoelectric micromachined modal gyroscope [15]. 


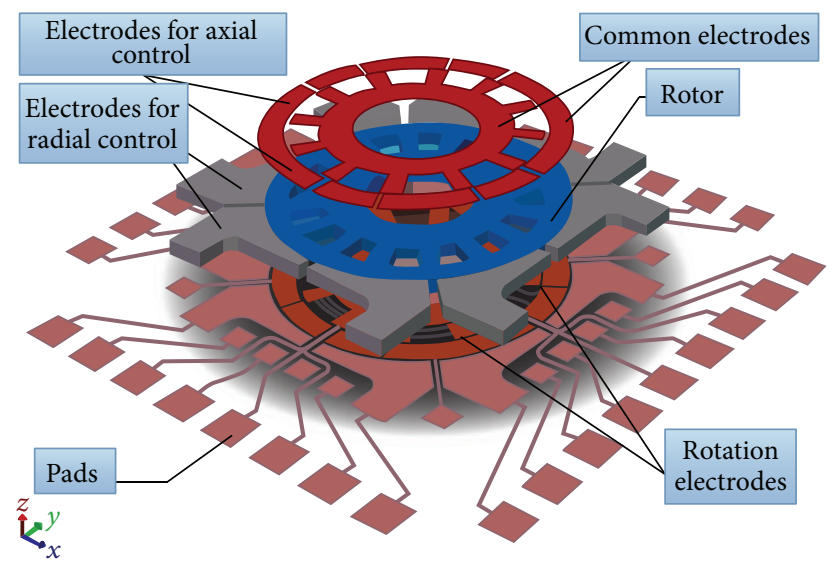

FIGURE 1: Maglev gyroscope.

As shown in Figure 1, it was suspended by the gyroscope's rotor that eliminates the machinery's friction and increases its accuracy. In 2003, Murakoshi et al. proposed the micromachined electrostatic suspension spinning gyroscope, Figure 1 [16]. Tsinghua University suggested a ring rotor gyroscope, Figure 2. The electric bearing of this gyroscope provides a noncontact suspension rotor, which can be slowly rotated in two orthogonal input shaft axes. It has an input range of $100 \pm 0.5^{\circ} \mathrm{s}^{-1}$, scaling factor of $39.8 \mathrm{mV} /\left({ }^{\circ} / \mathrm{s}^{-1}\right) \mathrm{Hz}$, and noise floor of $0.015^{\circ} \mathrm{s}^{-1} \mathrm{~Hz}^{-1 / 2}$. And the bias stability is $50.95^{\circ} / \mathrm{h}$ [17].

In recent years, the researchers from the University of Electronic Science and Technology in China investigated the LC tuning of magnetic suspension rotor gyroscope. This gyroscope mainly consists of a stator and a rotor. Its suspended rotor is driven, as shown in Figure 3. Magnetic suspension rotor gyroscope is electromagnetically suspended, with its rotor located at the center $[18,19]$.

In 2012, Tsinghua University and Harbin Industrial University investigated the rotor of liquid floated gyroscope. This gyroscope has high accuracy and mainly consists of a stator, rotor, coil, and test version. The edge of its structure is shown in Figure 4.

\section{Theory of Dished Rotor Gyroscope for Heat Coupling}

The heat coupling of gyroscope's rotor is produced by a magnetic field heat, heat flow field, and the composition of heat flow field and magnetic field coupling. The principle of these fields is shown in Figure 5. Dishing rotor gyroscope is produced by the stator coil in the magnetic dipole and is driven in a sealed cavity during high-speed rotation. The gyroscope is filled with \#3 industrial white oil in the rotating magnetic heated. The oil convection in the dished rotor is hot and it affects the precision of gyroscope measurements. The concrete principle is shown in Figure 5.

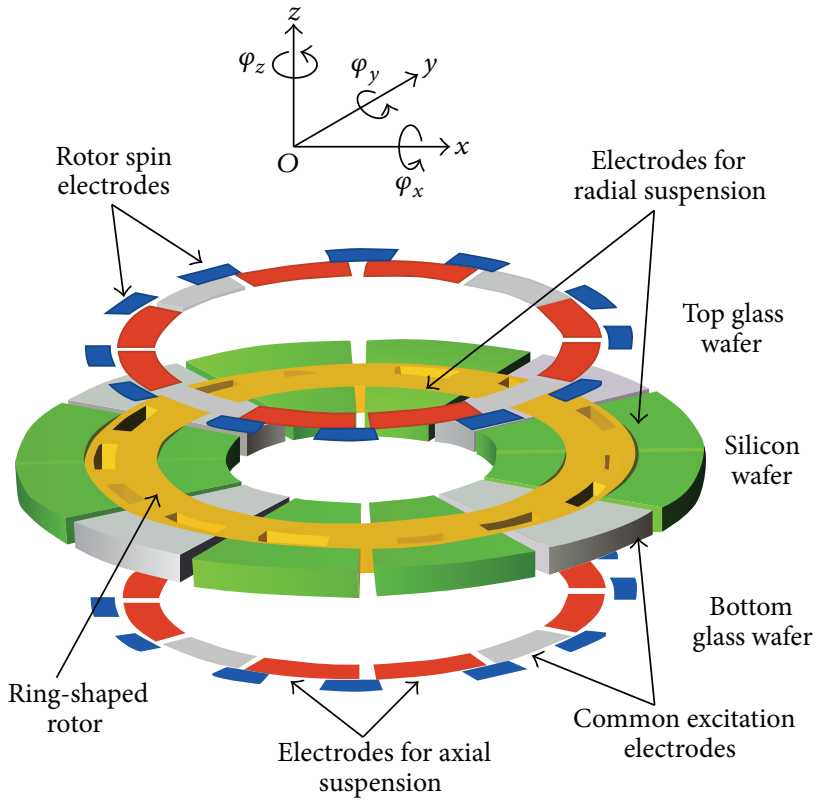

Figure 2: Tsinghua rotor gyroscopes.

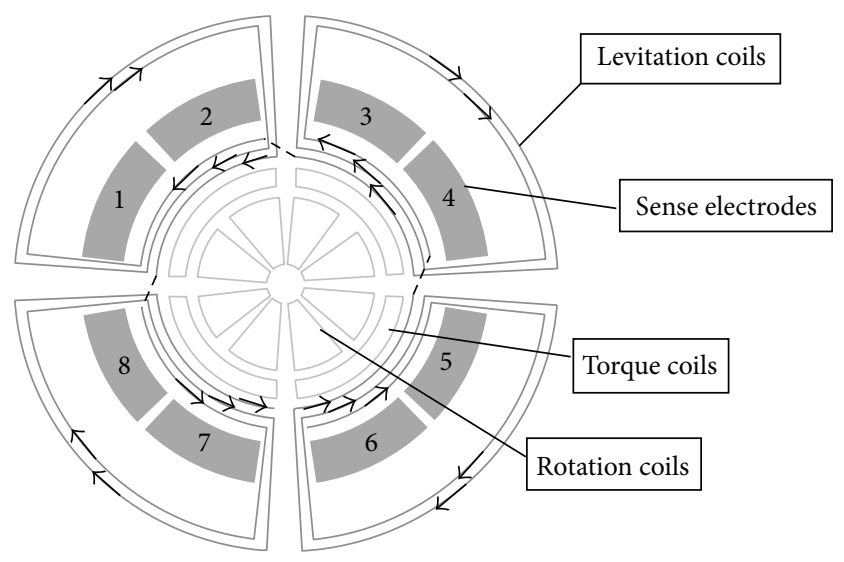

Figure 3: Rotor gyroscope.

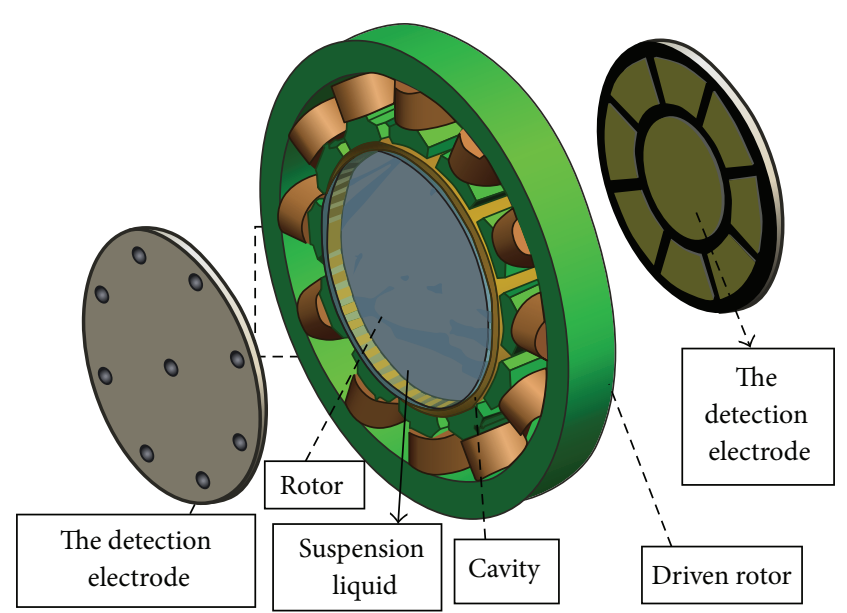

FIgURE 4: Maglev rotor gyroscopes. 


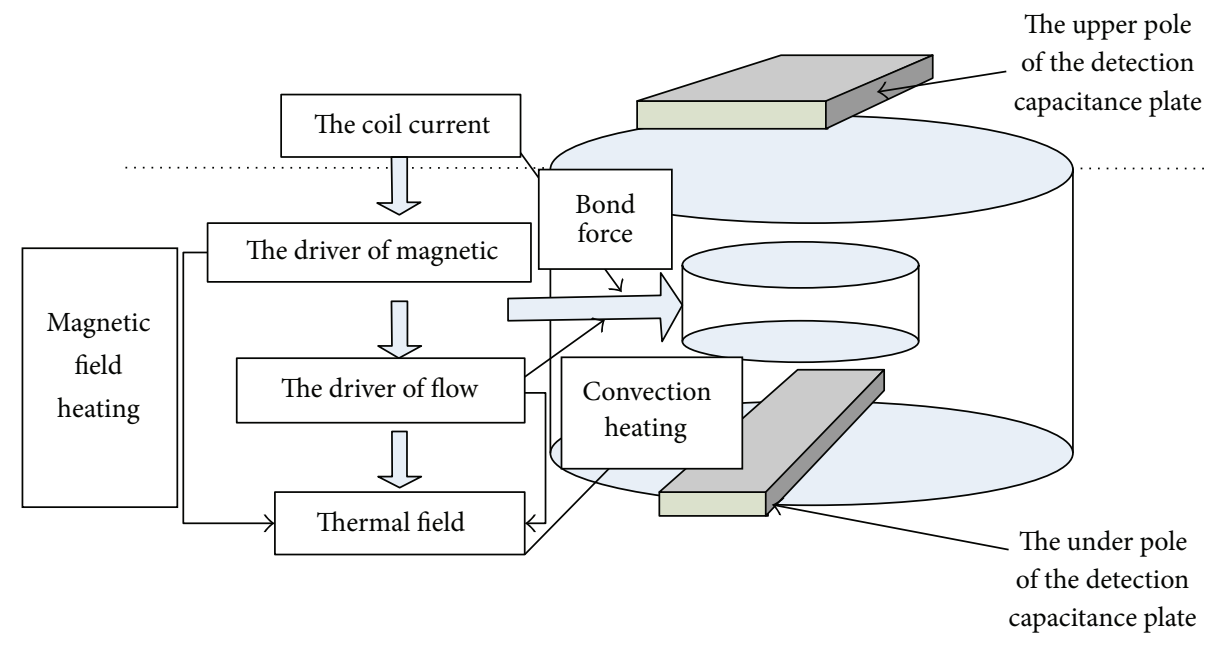

FIGURE 5: Coupling moment of rotor gyroscope.

2.1. Dynamic Torque Model of the Dishing Rotor Gyroscope. This is the moment of gyroscope equation:

$$
M^{\prime}=\left[\begin{array}{l}
M_{c i x}+M_{g x}+M_{f x}-\varphi_{i j x}-S_{i j x}-M_{t x}-\frac{D S}{d t}-\frac{d s}{d t} \\
M_{c i y}+M_{g y}+M_{f y}-\varphi_{i j y}-S_{i j y}-M_{t y}-\frac{D S}{d t}-\frac{d s}{d t} \\
M_{c i z}+M_{g z}-M_{f z}-\varphi_{i j z}-S_{i j z}-M_{t z}-\frac{D S}{d t}-\frac{d s}{d t}
\end{array}\right] .
$$

$M_{c i}$ is the driving moment of the stator coil magnetic field, $M_{g}$ is the dishing rotor by heavy torque, $M_{\text {floating } f z}$ is the float on the torque surface, $M_{\text {Float downward } f z}$ is the float on the down surface, $\varphi_{i j}$ is the pressure strain torque rotor of dishing \#3 industrial white oil, $S_{i j}$ is the torque produced by the rotation of \#3 industrial white oil, DS/ $d t$ is the circulating heat of the disc rotor (enthalpy) generated by \#3 industrial white oil, $d s / d t$ is the stator magnetic field (entropy) for the heat generated, and $M_{t}$ is the turbulence on the dished rotor torque.

The dynamic torque equation of the dishing rotor gyroscope in the stator coordinate system $\left(0-x_{2} y_{2} z_{2}\right)$ relative to the frame coordinate system $\left(0-x_{1} y_{1} z_{1}\right)$ is as follows:

$$
\begin{aligned}
& {\left[\begin{array}{l}
M_{x_{1}}^{\prime x_{2}} \\
M_{y_{1}}^{\prime y_{2}} \\
M_{z_{1}}^{\prime z_{2}}
\end{array}\right]} \\
& =\left(\begin{array}{ccc}
\cos \beta, & 0, & \sin \beta \\
0, & 1, & 0 \\
\sin \beta, & 0, & \cos \beta
\end{array}\right)\left[\begin{array}{c}
M_{x}^{\prime x_{1}} \\
M_{y}^{\prime y_{1}} \\
M_{z}^{\prime z_{1}}
\end{array}\right]
\end{aligned}
$$

$$
\begin{aligned}
= & \left(\begin{array}{ccc}
\cos \beta, & 0, & \sin \beta \\
0, & 1, & 0 \\
\sin \beta, & 0, & \cos \beta
\end{array}\right)\left(\begin{array}{ccc}
1, & 0, & 0 \\
0, & \cos \alpha, & -\sin \alpha \\
0, & \sin \alpha, & \cos \alpha
\end{array}\right) \\
& \times\left[\begin{array}{l}
M_{c i x}+M_{g x}+M_{f x}-\varphi_{i j x}-S_{i j x}-M_{t x}-\frac{D S}{d t}-\frac{d s}{d t} \\
M_{c i y}+M_{g y}+M_{f y}-\varphi_{i j y}-S_{i j y}-M_{t y}-\frac{D S}{d t}-\frac{d s}{d t} \\
M_{c i z}+M_{g z}-M_{f z}-\varphi_{i j z}-S_{i j z}-M_{t z}-\frac{D S}{d t}-\frac{d s}{d t}
\end{array}\right] .
\end{aligned}
$$

The dynamic torque equation of the dishing rotor gyroscope in the rotor coordinate system $\left(0-x_{R} y_{R} z_{R}\right)$ relative to the stator coordinate system $\left(0-x_{2} y_{2} z_{2}\right)$ is as follows:

$$
\left[\begin{array}{c}
M_{x_{2}}^{\prime x_{R}} \\
M_{y_{2}}^{\prime y_{R}} \\
M_{z_{2}}^{\prime z_{R}}
\end{array}\right]
$$

$$
\begin{aligned}
& =\left(\begin{array}{ccc}
\cos r, & -\sin r, & 0 \\
\sin r, & \cos r, & 0 \\
0, & 0, & 1
\end{array}\right)\left[\begin{array}{c}
M_{x_{1}}^{\prime x_{2}} \\
M_{y_{1}}^{\prime y_{2}} \\
M_{z_{1}}^{\prime z_{2}}
\end{array}\right] \\
& =\left(\begin{array}{ccc}
\cos r, & -\sin r, & 0 \\
\sin r, & \cos r, & 0 \\
0, & 0, & 1
\end{array}\right)\left(\begin{array}{ccc}
\cos \beta, & 0, & \sin \beta \\
0, & 1, & 0 \\
\sin \beta, & 0, & \cos \beta
\end{array}\right)
\end{aligned}
$$




$$
\begin{aligned}
& \times\left(\begin{array}{ccc}
1, & 0, & 0 \\
0, & \cos \alpha, & -\sin \alpha \\
0, & \sin \alpha, & \cos \alpha
\end{array}\right) \\
& \times\left[\begin{array}{l}
M_{c i x}+M_{g x}+M_{f x}-\varphi_{i j x}-S_{i j x}-M_{t x}-\frac{D S}{d t}-\frac{d s}{d t} \\
M_{c i y}+M_{g y}+M_{f y}-\varphi_{i j y}-S_{i j y}-M_{t y}-\frac{D S}{d t}-\frac{d s}{d t} \\
M_{c i z}+M_{g z}-M_{f z}-\varphi_{i j z}-S_{i j z}-M_{t z}-\frac{D S}{d t}-\frac{d s}{d t}
\end{array}\right] .
\end{aligned}
$$

The dynamic torque equation of the dishing rotor gyroscope in the stator coordinate system $\left(0-x_{2} y_{2} z_{2}\right)$ relative to the inertial coordinate system $(0-x y z)$ is as follows:

$$
\begin{aligned}
& {\left[\begin{array}{c}
M_{x}^{\prime x_{2}} \\
M_{y}^{\prime y_{2}} \\
M_{z}^{\prime z_{2}}
\end{array}\right]} \\
& =\left(\begin{array}{ccc}
\cos \beta, & 0, & \sin \beta \\
\sin \alpha \sin \beta, & \cos \alpha, & -\sin \alpha \cos \beta \\
-\cos \alpha \sin \beta, & \sin \alpha, & \cos \alpha \cos \beta
\end{array}\right) \\
& \times\left[\begin{array}{l}
M_{c i x}+M_{g x}+M_{f x}-\varphi_{i j x}-S_{i j x}-M_{t x}-\frac{D S}{d t}-\frac{d s}{d t} \\
M_{c i y}+M_{g y}+M_{f y}-\varphi_{i j y}-S_{i j y}-M_{t y}-\frac{D S}{d t}-\frac{d s}{d t} \\
M_{c i z}+M_{g z}-M_{f z}-\varphi_{i j z}-S_{i j z}-M_{t z}-\frac{D S}{d t}-\frac{d s}{d t}
\end{array}\right] .
\end{aligned}
$$

The dynamic torque equation of the dishing rotor gyroscope in the rotor coordinate system $\left(0-x_{R} y_{R} z_{R}\right)$ relative to the inertial coordinate system $(0-x y z)$ is as follows:

$$
\begin{aligned}
& {\left[\begin{array}{c}
M_{x}^{x_{R}} \\
M_{y}^{y_{R}} \\
M_{z}^{z_{R}}
\end{array}\right]=\left(\begin{array}{ccc}
\cos \beta \cos r, & -\cos \beta \sin r, & -\sin \beta \\
\cos \alpha \sin r+\sin \alpha \sin \beta \sin r, & \cos \alpha \cos r-\sin \alpha \sin \beta \sin r, & -\sin \alpha \cos \beta \\
\sin \alpha \sin r-\cos \alpha \sin \beta \cos r, & \sin \alpha \cos r+\cos \alpha \sin \beta \sin r, & \cos \alpha \cos \beta
\end{array}\right)} \\
& \times\left[\begin{array}{l}
M_{c i x}+M_{g x}+M_{f x}-\varphi_{i j x}-S_{i j x}-M_{t x}-\frac{D S}{d t}-\frac{d s}{d t} \\
M_{c i y}+M_{g y}+M_{f y}-\varphi_{i j y}-S_{i j y}-M_{t y}-\frac{D S}{d t}-\frac{d s}{d t} \\
M_{c i z}+M_{g z}-M_{f z}-\varphi_{i j z}-S_{i j z}-M_{t z}-\frac{D S}{d t}-\frac{d s}{d t}
\end{array}\right] .
\end{aligned}
$$

\subsection{Establishing the Dynamic Equations of the Dished Rotor} Gyroscope. In order to establish an equation about gyroscope torque, firstly, the hypothesis is as follows. The quality of the dishing rotor is represented by $m$ and its radius by $R$. The $x, y$, and $z$ axes in the coordinate system at the moment of the momentum are represented by $J_{x}, J_{y}, J_{z}$, respectively. The dishing rotor moment of the momentum $M_{x}, M_{y}, M_{z}$ represents the rotor moment of the momentum in the $x$, $y$, and $z$ axes of the direction projection. The setup $J, J_{1}$ represents the dishing rotor at the polar moment of the inertia moment and the equator. The moment of each coordinate system and the other parameters were previously mentioned. Through the moment of the momentum theorem is to the list of the dished rotor around 0 , and the Euler mechanics equation is as follows:

$$
\begin{aligned}
& J_{1}\left(\ddot{\beta}-\dot{\alpha}^{2} \cos \beta \sin \beta\right)+J \dot{\alpha} \sin \beta(\dot{r}+\dot{\alpha} \cos \beta)=M_{x}, \\
& J_{1}(\ddot{\alpha} \sin \beta+2 \dot{\alpha} \dot{\beta} \cos \beta)-J \dot{\beta}(\dot{r}+\dot{\alpha} \cos \beta)=M_{y}, \\
& J \frac{d}{d t}(r+\dot{\alpha} \cos \beta)=M_{z} .
\end{aligned}
$$

The $z$ and $x$ axes are the polar and equatorial axes of the dishing rotor, respectively. Given that $J=J_{z}, J_{1}=J_{x}$, the rotor's origin was set, and its name was $Q$. It is $O \vec{Q}\left(x_{0}, y_{0}, z_{0}\right)$, and its cosine is $O \vec{Q}\left(x_{0}, y_{0}, z_{0}\right)$. Meanwhile, it is relative to the inertial coordinate system as follows: $i, j, k$, and the moment of inertia of the dishing rotor is as follows:

$$
J_{\mathrm{QQ}}=\int\left[x_{0}^{2}+y_{0}^{2}+x_{0}^{2}-\left(x_{0} \alpha+y_{0} \beta+z_{0} r\right)^{2}\right] d m \text {. }
$$

Therefore, there are

$$
\begin{gathered}
J=J_{z}=\int\left(x_{0}^{2}+y_{0}^{2}\right) d m, \\
J_{1}=J_{x}=\int\left(y_{0}^{2}+z_{0}^{2}\right) d m .
\end{gathered}
$$

The front dished rotor about the equation of mechanical characteristics is calculated as follows:

$$
J_{1}\left(\ddot{\beta}-\dot{\alpha}^{2} \cos \beta \sin \beta\right)+J \dot{\alpha} \sin \beta(\dot{r}+\dot{\alpha} \cos \beta)=M_{x},
$$




$$
\begin{aligned}
& J_{1}(\ddot{\alpha} \sin \beta-2 \dot{\alpha} \dot{\beta} \cos \beta)+J \dot{\beta}(\dot{r}+\dot{\alpha} \cos \beta)=M_{y}, \\
& J \frac{d}{d t}(r+\dot{\alpha} \cos \beta)=M_{z} .
\end{aligned}
$$

The dished rotor moves at a high-speed in a closed chamber filled with \#3 industrial white oil. The rotor is in the $x$ - and $y$ - axes with deflection angles $\alpha, \beta$. Thus, it can be approximated.

The dynamic equation is simplified. Consider

$$
\begin{gathered}
J_{1} \ddot{\beta}=M_{x}, \\
2 J_{1} \dot{\alpha} \dot{\beta}-J \dot{\beta}(\dot{r}+\dot{\alpha})=M_{y}, \\
J \frac{d}{d t}(r+\dot{\alpha})=M_{z} .
\end{gathered}
$$

Solving (10), the following equations are obtained:

$$
\begin{aligned}
& \dot{\beta}=\frac{M_{x} t}{J_{1}}+\frac{C_{1}}{J_{1}}, \\
& \beta=\frac{1}{2} \frac{M_{x} t^{2}}{J_{1}}+\frac{C_{1}}{J_{1}} t+C_{2} \text {, } \\
& \dot{\alpha}=\frac{M_{y}}{\left[2 J_{1}-\left(M_{z} / J\right) t+C_{3}\right]\left[\left(M_{x} / J_{1}\right) t+C_{1} / J_{1}\right]}, \\
& \alpha=\frac{M_{y}}{\left[2 J_{1}-\left(M_{z} / J\right) t+C_{3}\right]\left[\left(M_{x} / J_{1}\right) t+C_{1} / J_{1}\right]} t+C_{4}, \\
& r=\frac{M_{z}}{J} t+C_{3}-\dot{\alpha} \\
& =\frac{M_{z}}{J} t+C_{3} \\
& -\frac{M_{y}}{\left[2 J_{1}-\left(M_{z} / J\right) t+C_{3}\right]\left[\left(M_{x} / J_{1}\right) t+C / J_{1}\right]}, \\
& \dot{r}=\frac{M_{z}}{J}-\ddot{\alpha}, \\
& \dot{r}=\frac{M_{z}}{J}\left(-\frac{M_{y} M_{z}}{J_{1}}\left[M_{x} t+C_{1}\right] \frac{1}{\left(2 J_{1}-\left(M_{z} / J\right) t+C_{3}\right)^{2}}\right. \\
& +\frac{M_{x} M_{y}}{J_{1}\left(2 J_{1}-\left(M_{z} / J\right) t+C_{3}\right)} \\
& \left.\times \frac{1}{\left(M_{x} t / J_{1}+C_{1} / J_{1}\right)^{2}}\right) .
\end{aligned}
$$

2.3. Dynamic Equation of Generation of the Dished Rotor Gyroscope. The dynamic equation (6) is substituted to the relevant torque as follows:

$M_{x}=\cos \beta \cos r$

$$
\begin{aligned}
& \times\left(M_{e x}+M_{g x}+M_{f x}-\varphi_{i j x}-S_{i j x}-M_{t x}\right. \\
& \left.-\frac{D S}{d t}-\frac{d s}{d t}\right) \\
& -\cos \beta \sin r \\
& \times\left(M_{c i y}+M_{g y}+M_{f y}-\varphi_{i j y}-S_{y}-M_{t y}-\frac{D S}{d t}-\frac{d s}{d t}\right) \\
& -\sin \beta\left(M_{c i z}+M_{g z}+M_{f z}-\varphi_{i j z}-S_{z}-M_{t z}\right. \\
& \left.\quad-\frac{D S}{d t}-\frac{d s}{d t}\right),
\end{aligned}
$$

$M_{y}=(\cos \alpha \sin r+\sin \alpha \sin \beta \sin r)$

$$
\begin{aligned}
& \times\left(M_{c i x}+M_{g x}+M_{f x}-\varphi_{x}-S_{x}-M_{t x}-\frac{D S}{d t}-\frac{d s}{d t}\right) \\
& +(\cos \alpha \cos r-\sin \alpha \sin \beta \sin r) \\
& \times\left(M_{c i y}+M_{g y}+M_{f y}-\varphi_{y}-S_{y}-M_{t y}-\frac{D S}{d t}-\frac{d s}{d t}\right)
\end{aligned}
$$$$
-\sin \alpha \cos \beta
$$$$
\times\left(M_{c i z}+M_{g z}+M_{f z}-\varphi_{z}-S_{z}-M_{t z}-\frac{D S}{d t}-\frac{d s}{d t}\right),
$$$$
M_{z}=(\sin \alpha \sin r+\cos \alpha \sin \beta \sin r)
$$$$
\times\left(M_{c i x}+M_{g x}+M_{f x}-\varphi_{x}-S_{x}-M_{t x}-\frac{D S}{d t}-\frac{d s}{d t}\right)
$$$$
+(\sin \alpha \cos r-\cos \alpha \sin \beta \sin r)
$$$$
\times\left(M_{c i y}+M_{g y}+M_{f y}-\varphi_{y}-S_{y}-M_{t y}-\frac{D S}{d t}-\frac{d s}{d t}\right)
$$$$
-\cos \alpha \cos \beta
$$$$
\times\left(M_{c i z}+M_{g z}+M_{f z}-\varphi_{z}-S_{z}-M_{t z}-\frac{D S}{d t}-\frac{d s}{d t}\right) .
$$

At the same time,

$$
\begin{aligned}
& J=J_{z}=\int\left(x_{0}^{2}+y_{0}^{2}\right) d m, \\
& J_{1}=J_{x}=\int\left(y_{0}^{2}+z_{0}^{2}\right) d m .
\end{aligned}
$$

Substituting (18) into (11), (13), and (16), the following equations are obtained. 
At the same time, (19) was calculated by the torque, and the angular velocity is as follows:

$$
\begin{gathered}
\dot{\alpha}=\frac{M_{y}}{\left[2 J_{1}-\left(M_{z} / J\right) t+C_{3}\right]\left[\left(M_{x} / J_{1}\right) t+C_{1} / J_{1}\right]}, \\
\dot{\beta}=\frac{M_{x} t}{J_{1}}+\frac{C_{1}}{J_{1}}, \\
\dot{r}=\frac{M_{z}}{J}-\left(-\frac{M_{y} M_{z}}{J_{1}}\left[M_{x} t+C_{1}\right] \frac{1}{\left(2 J_{1}-\left(M_{z} / J\right) t+C_{3}\right)^{2}}\right. \\
\quad+\frac{M_{x} M_{y}}{J_{1}\left(2 J_{1}-\left(M_{z} / J\right) t+C_{3}\right)} \\
\left.\quad \times \frac{1}{\left(M_{x} t / J_{1}+C_{1} / J_{1}\right)^{2}}\right) .
\end{gathered}
$$

These three equations are angular velocity calculated by coupling torque about this kind of liquid floated gyroscope. At the same time, (20) were substituted into the moment. To calculate the reduction of the angular velocity, the following are assumed:

$$
\begin{array}{lll}
\cos \alpha=1, & \cos \beta=1, & \cos r=1, \\
\sin \alpha=\alpha, & \sin \beta=\beta, & \sin r=r .
\end{array}
$$

Simplifying the equation yields

$$
\begin{aligned}
& M_{x x}=M_{c i x}+M_{g x}+M_{f x}-\varphi_{x}-S_{x}-M_{t x}-\frac{D S}{d t}-\frac{d s}{d t}, \\
& M_{y y}=M_{c i y}+M_{g y}+M_{f y}-\varphi_{y}-S_{y}-M_{t y}-\frac{D S}{d t}-\frac{d s}{d t}, \\
& M_{z z}=M_{c i z}+M_{g z}+M_{f z}-\varphi_{z}-S_{z}-M_{t z}-\frac{D S}{d t}-\frac{d s}{d t} .
\end{aligned}
$$

Equation (23) is substituted into the above equation:

$$
\begin{gathered}
\dot{\alpha}=\left((r+\alpha \beta r) M_{x x}+(1-\alpha \beta r) M_{y y}-\alpha \beta M_{z z}\right) \\
\times\left[2 \int\left(y_{0}^{2}+z_{0}^{2}\right) d m\right. \\
\left.\quad-\frac{(\alpha r-\beta) M_{x x}+(\alpha+\beta r) M_{y y}+M_{z z}}{\int\left(x_{0}^{2}+y_{0}^{2}\right) d m} t+C_{3}\right]^{-1} \\
\times\left[\int \frac{M_{x x}+(1-\alpha \beta r) M_{y y}-\alpha M_{z z}}{\int\left(y_{0}^{2}+z_{0}^{2}\right) d m} t\right. \\
\dot{\beta}=\frac{C_{x x}-r M_{y y}-\alpha M_{z z}}{\int\left(y_{0}^{2}+z_{0}^{2}\right) d m} t+\frac{C_{1}}{\int\left(y_{0}^{2}+z_{0}^{2}\right) d m}
\end{gathered}
$$

$$
\begin{aligned}
\dot{r}= & \frac{(\alpha r-\beta) M_{x x}-(\alpha+\beta r) M_{y y}+M_{z z}}{\int\left(x_{0}^{2}+y_{0}^{2}\right) d m} \\
& +\frac{(\alpha r-\beta r) M_{x x}-(\alpha+\beta r) M_{y y}+M_{z z}}{\int\left(z_{0}^{2}+y_{0}^{2}\right) d m}
\end{aligned}
$$$$
\times\left((r+\alpha \beta r) M_{x x}+(\alpha+\beta r) M_{y y}+M_{z z}\right)
$$$$
\times\left(\left(M_{x x}+(1+\alpha \beta r) M_{y y}+M_{z z}+C_{1}\right)\right.
$$$$
\times\left(\left(2 \int\left(y_{0}^{2}+z_{0}^{2}\right) d m\right.\right.
$$$$
-\frac{(\alpha r-\beta) M_{x x}+(\alpha+\beta r) M_{y y}+M_{z z}}{\int(x+y) d m} t
$$$$
\left.\left.+C_{3}\right)^{2}\right)^{-1}
$$$$
+\left((\alpha r+\beta r) M_{x x}+(\alpha+\beta r) M_{y y}+M_{z z}\right)
$$$$
\times\left((r+\alpha \beta r) M_{x x}+(1-\alpha \beta r) M_{y y}+\alpha M_{z z}\right)
$$$$
\times\left(2 \int\left(y_{0}^{2}+z_{0}^{2}\right) d m\right.
$$$$
-\frac{(\alpha r-\beta) M_{x x}+(\alpha+\beta r) M_{y y}+M_{z z}}{\int\left(x_{0}^{2}+y_{0}^{2}\right) d m} t
$$$$
\left.+C_{3}\right)^{-1}
$$$$
\times\left(\left(\int \frac{M_{x x}+r M_{y y}+\alpha M_{z z}}{\int\left(x_{0}^{2}+y_{0}^{2}\right) d m} t\right.\right.
$$

$$
\left.\left.+\frac{C}{\int\left(y_{0}^{2}+z_{0}^{2}\right)}\right)^{2}\right)^{-1}
$$

2.4. Theory of Hot Fluid about the Gyroscope. For the theory coupled thermal field of dished rotor, these are as follows. First is the coupling thermodynamics calculation; second is the establishment of boundary conditions.

2.4.1. Coupled Thermodynamic Theory of the Gyroscope's Rotor. The volume of the dishing rotor is assumed to consist of two parts: spherical and cylindrical. The volume of the sphere is calculated as follows:

$$
V=V_{1}+V_{2}=\frac{4}{3} \pi\left(r_{2}^{3}-r_{1}^{3}\right)+h \pi\left(r_{3}^{2}-r_{2}^{2}\right) .
$$


The whole dishing rotor is in the thermal field, and the temperature is simulated. Consider

$$
\begin{aligned}
T= & \frac{g_{0}}{3 k}\left[\frac{r_{2}^{2}-r^{2}}{2}-r_{1}^{3}\left(\frac{1}{r}-\frac{1}{r_{2}}\right)+\frac{r_{2}}{h}\left(1-\frac{r_{1}^{3}}{r_{2}^{3}}\right)\right]+60 \\
& +\frac{1}{r} \sum_{m=1}^{\infty}\left[\frac{1}{N\left(\beta_{m}\right)} \int_{r_{1}}^{r_{2}} X\left(\beta_{m}, r\right) r\left(T_{0}-T_{a 0}\right) d r\right] \\
& \times X(\beta, r) e^{-\alpha \beta_{m}^{2} t} \\
& +\frac{g_{0}}{3 k}\left[\frac{r_{3}^{2}-r^{\prime 2}}{2}-r_{2}^{3}\left(\frac{1}{r^{\prime}}-\frac{1}{r_{3}}\right)+\frac{r_{3}}{h}\left(1-\frac{r_{2}^{3}}{r_{3}^{3}}\right)\right]+60 \\
& +\frac{1}{r^{\prime}} \sum_{m=1}^{\infty}\left[\frac{1}{N\left(\beta_{m}\right)} \int_{r_{2}}^{r_{3}} X\left(\beta_{m}, r^{\prime}\right) r^{\prime}\left(T_{0}-T_{a 0}\right) d r^{\prime}\right] \\
& \times X\left(\beta, r^{\prime}\right) e^{-\alpha \beta_{m}^{2} t} .
\end{aligned}
$$

The equation above can be reduced. The following was established and the dished rotor was in coupled thermal field about the calculation of volume and temperature. Consider

$$
\begin{aligned}
& T=\frac{g_{0}}{3 k}\left[\frac{r_{2}^{2}+r_{3}^{2}-\left(r^{2}-r^{\prime 2}\right)}{2}-r_{1}^{3}\left(\frac{1}{r}+\frac{1}{r^{\prime}}-\frac{1}{r_{3}}-\frac{1}{r_{2}}\right)\right. \\
&\left.+\frac{r_{2}}{h}\left(1-\frac{r_{1}^{3}}{r_{2}^{3}}\right)+\frac{r_{3}}{h}\left(1-\frac{r_{2}^{3}}{r_{3}^{3}}\right)\right] \\
&+120+\frac{1}{r} \sum_{m=1}^{\infty}\left[\frac{1}{N\left(\beta_{m}\right)} \int_{r_{1}}^{r_{2}} X\left(\beta_{m}, r\right) r\left(T_{0}-T_{a 0}\right) d r\right] \\
& \times X(\beta, r) e^{-\alpha \beta_{m}^{2} t} .
\end{aligned}
$$

The thermodynamic analysis of the dishing rotor gyroscope shows a continuous connection between the wellposed equation of differential thermodynamics and the continuum mechanics of the problems, because the dishing rotor gyroscope is filled with \#3 industrial white oil; thus, a Godunov-type equation can be applied [20]:

$$
\begin{gathered}
\frac{\partial s_{p}^{*}}{\partial \tau}=-\operatorname{div} g_{p}, \\
\frac{\partial\left(p_{k} s_{p k}^{*}-s^{*}\right)}{\partial \tau}=-\frac{\partial\left(p_{k} g_{p k}^{j}-g^{j}\right)}{\partial r_{j}} .
\end{gathered}
$$

This gyroscope operates under high-speed rotation: a form of nonequilibrium thermodynamics and state variables can be established as follows:

$$
\begin{aligned}
\frac{\partial f(x, y, z)}{\partial \tau}= & -\frac{\partial}{\partial x}\left(f(x, y, z) L \Phi_{x}(x, y, z)\right) \\
& +\frac{\partial}{\partial x}\left(\Lambda f(x, y, z) \Phi_{x}(x, y, z)\right)
\end{aligned}
$$

$$
\begin{aligned}
\frac{\partial f(x, y, z)}{\partial \tau}= & -\frac{\partial}{\partial y}\left(\Lambda k_{B} \frac{\partial f(x, y, z)}{\partial x}\right), \\
& +\frac{\partial}{\partial y}\left(\Lambda f(x, y, z) \Phi_{y}(x, y, z)\right) \\
& +\frac{\partial}{\partial y}\left(\Lambda k_{B} \frac{\partial f(x, y, z)}{\partial y}\right), \\
\frac{\partial f(x, y, z)}{\partial \tau}= & -\frac{\partial}{\partial z}\left(f(x, y, z) L \Phi_{z}(x, y, z)\right) \\
& +\frac{\partial}{\partial z}\left(\Lambda f(x, y, z) \Phi_{z}(x, y, z)\right) \\
& +\frac{\partial}{\partial z}\left(\Lambda k_{B} \frac{\partial f(x, y, z)}{\partial z}\right) .
\end{aligned}
$$

Among them,

$$
\Phi(f)=\int d x f(x)\left(\Phi(x)+k_{B} \ln f(x)\right) .
$$

The Boltzmann equation of thermodynamics of the dishing rotor heat entropy in the growth equation is as follows [20]:

$$
\begin{aligned}
& \{A, B\}^{(0)} \\
& =\int d r \int d v \\
& \times\left[\int f\left(\partial_{i}\left(A_{f}\right) \frac{\partial}{\partial v_{i}}\left(B_{f}\right)-\partial_{i}\left(B_{f}\right) A_{F_{i}(1)}\right)\right. \\
& +\eta\left(\partial_{i}\left(A_{F_{i}(1)}\right) \frac{\partial}{\partial v_{i}}\left(B_{f}\right)-\partial_{i}\left(B_{s}\right) \frac{\partial\left(A_{f}\right)}{\partial v_{i}}\right) \\
& +f\left(\partial_{i}\left(A_{F(0)}\right) \frac{\partial}{\partial v_{i}}\left(B_{f}\right)\right) \\
& \left.-\partial_{i}\left(B_{F(0)} \frac{\partial}{\partial v_{i}}\left(A_{f}\right)\right)\right], \\
& \frac{\partial f(r, v)}{\partial t}=-\partial_{i}\left(f \frac{\partial \Phi_{f}}{\partial v_{i}}\right) \\
& +\frac{\partial}{\partial v_{i}}\left(f \partial_{i} \Phi_{f}+\eta \partial_{i} \Phi_{s}+f \partial_{i} \Phi_{F(0)}\right), \\
& \frac{\partial s}{\partial t}=-\partial_{i} \int d v \eta \frac{\partial \Phi_{f}}{\partial v_{i}}, \\
& \frac{\partial F^{(0)}}{\partial t}=-\partial_{i} \int d v f \frac{\partial \Phi_{f}}{\partial v_{i}} .
\end{aligned}
$$


The medium oil film of the dishing rotor gyroscope is produced by a model of the turbulent flow of inhomogeneous superfluid thermodynamics. The specific liquid properties of \#3 industrial white oil are given. The average vortex line length is measured in unit volume and heat flux restructuring expression. The second principle of thermodynamics is combined with the Lagrange equation using the Legendre transformation through the constitutive theory. The complete expression of nonequilibrium and its entropy flux has been established [21], and the constitutive relation is as follows:

$$
\begin{aligned}
& \frac{\partial \rho}{\partial t}+\frac{\partial \rho v_{j}}{\partial x_{j}}=0, \\
& \frac{\partial \rho v_{i}}{\partial t}+\frac{\partial}{\partial x_{j}}\left(\rho v_{i} v_{j}+J_{i j}^{v}\right)=0, \\
& \frac{\partial}{\partial t}\left(E+\frac{1}{2} \rho v^{2}\right)+\frac{\partial}{\partial x_{j}}\left[v_{j}\left(E+\frac{1}{2} \rho v^{2}\right)+v_{i} J_{i j}^{v}+q_{j}\right]=0, \\
& \frac{\partial m_{i}}{\partial t}+\frac{\partial}{\partial x_{j}}\left(m_{i} v_{j}+J_{i j}^{m}\right)=Q_{i}^{m}, \\
& \frac{\partial L}{\partial t}+\frac{\partial}{\partial x_{j}}\left(L v_{j}+J_{j}^{L}\right)=Q^{L} .
\end{aligned}
$$

This is a part of the calculated heat about dished rotor, where $v$ represents the average velocity, $\rho$ is density, $v_{i}$ is velocity, $E$ is energy density, $m_{i}$ is an internal variable, and $L$ is the density of the vortex line [22].

The turbulence theory of nonlinear superfluid involves entropy flux with different temperatures and heat flux of the dishing rotor [21]. The complete expression of the nonequilibrium entropy flux is as follows:

$$
\Phi_{i}=\left(\frac{1}{\theta}+v \Lambda_{L}+\gamma \lambda q^{2}\right) q_{i}=\frac{1}{\theta}\left(q_{i}-u_{L} J_{i}^{L}+\theta_{\gamma} \lambda q^{2} q_{i}\right)
$$

The thermodynamic analysis for the oil film is performed on the isothermal surface [23].

In thermodynamic analysis, the lattice Boltzmann method is successfully applied in various problems related to isothermal fluid dynamics [24]. However, the application of the method in nonisothermal problems is limited; the heat model causes numerical instability [25]. The thermal lattice Boltzmann model can generally be divided into two types [26]. The first type is the multispeed model, and the second type is the passive scalar. The main advantage of the passive scalar model over the multispeed model is that the former enhances numerical stability; thus, the stability of the gyroscope must be ensured to enhance the hybrid finite thermal model in the difference method [27, 28]. Applying the thermal lattice Boltzmann equation in the gyroscope has been proposed [29]. The model of uniform lattice BGK collision must be adopted; it can be expressed as follows:

$$
\begin{aligned}
& f_{i}\left(\vec{x}+\vec{e}_{i} \Delta t, t+\Delta t\right)=f_{i}(\vec{x}, t)-\frac{1}{\tau_{f}}\left[f_{i}(\vec{x}, t)-f_{i}^{\mathrm{eq}}(\vec{x}, t)\right], \\
& g_{i}\left(\vec{x}+\vec{e}_{i} \Delta t, t+\Delta t\right)=g_{i}(\vec{x}, t)-\frac{1}{\tau_{g}}\left[g_{i}(\vec{x}, t)-g_{i}^{\mathrm{eq}}(\vec{x}, t)\right],
\end{aligned}
$$

where $f_{i}$ and $g_{i}$ are the particle density and the function energy distribution, particularly of the particle velocity direction $e . \tau_{f}$ and $\tau_{g}$ represent the dimensionless relaxation time, whose control interest rates are close to the state of balance. Consider

$$
\begin{gathered}
\rho=\sum_{i} f_{i}, \\
\rho \vec{u}=\sum_{i} f_{i} \vec{e}_{i}, \\
\rho \frac{1}{2} D_{0} R T=\sum_{i} g_{i} .
\end{gathered}
$$

Balance the function of density distribution for these two models. Consider

$$
f_{i}^{\mathrm{eq}}=w_{i} \rho\left[1+\frac{3 \vec{e}_{i} \cdot \vec{u}}{C^{2}}+\frac{9\left(\vec{e}_{i} \cdot \vec{u}\right)^{2}}{2 C^{4}}-\frac{3 \vec{u} \cdot \vec{u}}{2 C^{2}}\right] .
$$

These are the coupled thermodynamic theory of gyroscope's rotor.

2.4.2. Boundary Conditions of the Dished Rotor Gyroscope in Fluid Dynamics. The boundary of the dished rotor gyroscope was assumed to be in the \#3 industrial white oil. The function of the particle density distribution is expressed as follows:

$$
f_{i}(\vec{x}, t)=f_{i}^{*}(\vec{x}, t)+\frac{w_{i}}{C} \vec{e}_{i} \cdot \vec{Q} .
$$

A pressure oil body exists but is applicable only along the edge of the density distribution function. As proposed by Fermi [30], the boundary conditions of \#3 industrial white oil velocities can be determined as follows:

$$
\begin{aligned}
\rho= & f_{0}+\left[f_{1}^{*}+w_{1} Q_{x}\right]+f_{2}+f_{3}+f_{4} \\
& +\left[f_{5}^{*}+w_{5}\left(Q_{x}+Q_{y}\right)\right]+f_{6}+f_{7} \\
& +\left[f_{8}^{*}+w_{8}\left(Q_{x}-Q_{y}\right)\right], \\
\rho u= & {\left[f_{1}^{*}+w_{1} Q_{x}\right]+\left[f_{5}^{*}+w_{5}\left(Q_{x}+Q_{y}\right)\right] } \\
& +\left[f_{8}^{*}+w_{8}\left(Q_{x}-Q_{y}\right)\right]-f_{3}-f_{6}-f_{7}, \\
\rho v= & f_{2}+\left[f_{1}^{*}+w_{5}\left(Q_{x}+Q_{y}\right)\right]+f_{6}-f_{4}-f_{7} \\
& -\left[f_{8}^{*}+w_{8}\left(Q_{x}-Q_{y}\right)\right] .
\end{aligned}
$$




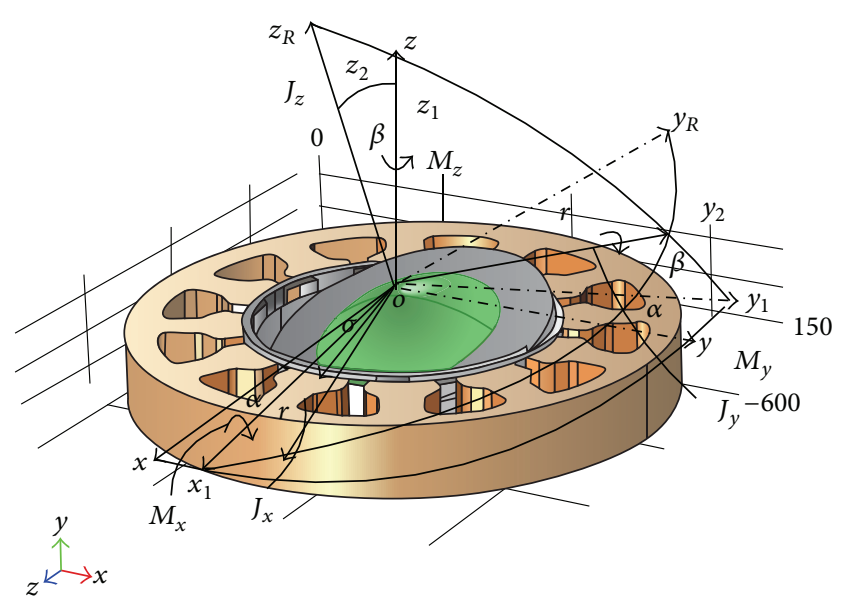

Figure 6: Dynamic torque of liquid floated gyroscope.

The common thermodynamic framework extensive key depends on the assumption of entropy [30]. Some of the common thermodynamic relations, including Claudius thermodynamic entropy, heat, and entropy, are as shown in Figure 6. The theoretical formula is also suitable for dished rotor about the liquid floated gyroscope [31]:

$$
S_{q}(A, B)=S_{q}(A)+S_{q}(B)+\frac{1-q}{k} S_{q}(A) S_{q}(B) .
$$

\section{Thermodynamic Simulation of the Dished Rotor}

The thermodynamic theory of simulation for dished rotor, we demonstrate respectively from two aspects of $2 \mathrm{D}$ and $3 \mathrm{D}$ simulation.

3.1. 2D Simulation of the Thermodynamics of the Dished Rotor Gyroscope in the COMSOL. When the dishing rotor gyroscope is in a confined space, the pressure of the fluid in the COMSOL is simulated, particularly the temperature field of the gyroscope's rotor. It can be considered by the rotor flow field; these are for the gyroscope that can produce the Eddy current. The temperature of this gyroscope's rotor is high. In the COMSOL simulation, the inner cavity joins the laminar flow in the four corners of the stator. The maximum temperature is shown in Figure 7(a). When the rotor is operating at $5549 \mathrm{r} / \mathrm{min}$, the turbulent flow velocity of the simplified version of the gyroscope increases the turbulence, particularly the inner cavity (Figure 17). At this point, the rotor reaches the highest temperature, indicating the occurrence of the phenomenon of chaos (Figure $7(\mathrm{~b})$ ). Thus, the rotor must be processed under the same conditions shown in Figure 7(b), where the temperature and the phenomenon of laminar flow decrease, particularly the temperature of the plate above the board.

3.2. 3D Simulation of the Thermodynamics of the Dished Rotor Gyroscope in the COMSOL. The coupled thermal simulation of the dishing rotor and heat coupling were performed in the 3D simulation of COMSOL. They were conducted using a grid, as shown in Figure 8.

The rotor's coupling heat was simulated with the gyroscope's rotor speeds of $5000 \mathrm{rad} / \mathrm{min}$ and $5549 \mathrm{rad} / \mathrm{min}$, and the results are shown in Figures 9(a) and 9(b).

3.2.1. Simulation Measurement of the Dished Rotor Gyroscope. The coupling force field of the dishing rotor gyroscope and the rotor's coupling heat were simulated with the gyroscope's rotor speeds of 5000 and $5549 \mathrm{rad} / \mathrm{min}$, as shown in Figures 10 (a) and $10(\mathrm{~b})$.

The rotor is driven by a magnetic field, and the gyroscope's rotor speeds are 5000 and $5549 \mathrm{rad} / \mathrm{min}$. The analysis of the thermal coupling characteristic curve is shown in Figures 11(a) and 11(b). One edge of the rotor is in the largest thermal field force and has a maximum temperature of $83.25^{\circ}$. Thus, the size of the rotor thermal coupling must be improved to precisely enhance the detection of the gyroscope. This improvement can be achieved by two methods. First, the rotor boundary is processed into $0.005 \mathrm{~mm}$ radian; second, the rotor is processed using nanomaterials

3.3. Moment of the Dished Rotor Gyroscope in ADAMS Simulation. ADAMS has established a model of the liquid floated gyroscope. A grid should be constructed for the gyroscope during the simulation of the dishing rotor. The gyroscope's rotor is placed in the grid, as shown in Figure 12.

The simulation model is established by measuring the rotation of the dishing rotor, particularly its angular velocity and the force and speed of the gyroscope's rotor. The results are shown in Figure 13. Therefore, we need to process the dished rotor.

\section{The Heat Thermal Experiment about the Thermal Fluid of the Dished Rotor}

This experiment adopts the phase field equation that can be derived from the extremum principle of thermodynamics, which describes the state of the system. The quantity can be obtained by applying the extremum principle model for thermodynamics and the evolution of the constitutive equation for the thermodynamic system. The phase field method is a potential simulation tool. The microstructure evolution of a system is complex, and several parameters are introduced as the standards in thermodynamics. The relationship between thermodynamics and phase field parameters is analyzed, simulated, and verified [32].

The total Gibbs energy of the GS mechanochemical system is given by

$$
\begin{aligned}
G_{S} & =\int_{V} g_{s} d V \\
& \approx \Delta \sum_{x=1}^{X}\left(w^{\alpha}(\phi(x)) g^{\alpha}+w^{\beta}(\phi(x)) g^{\beta}+w^{I}(\phi(x)) g^{I}\right) .
\end{aligned}
$$




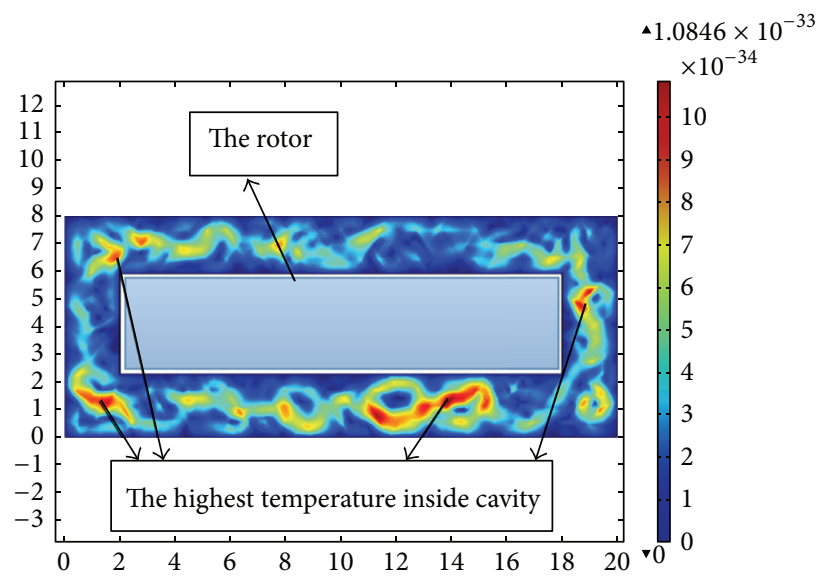

(a)

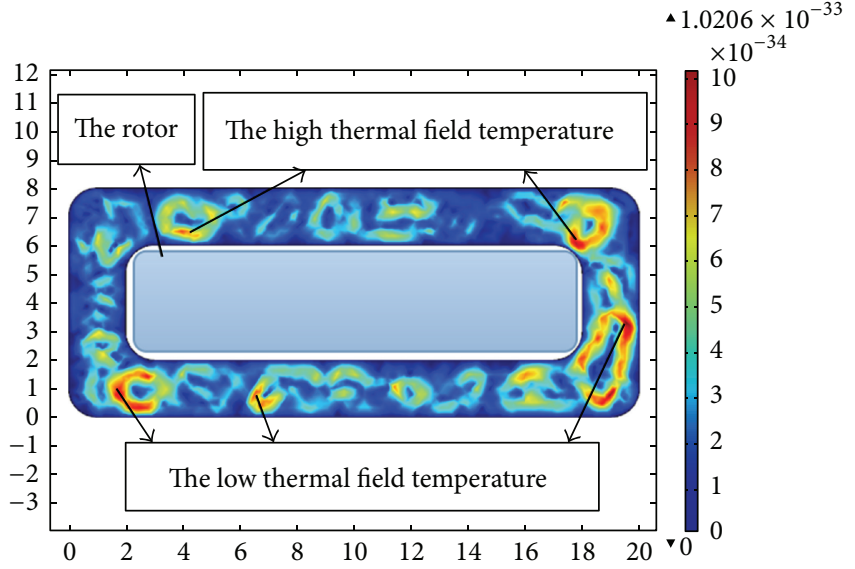

(b)

Figure 7: Thermal field of temperature under different rotor structures ((a), (b)).

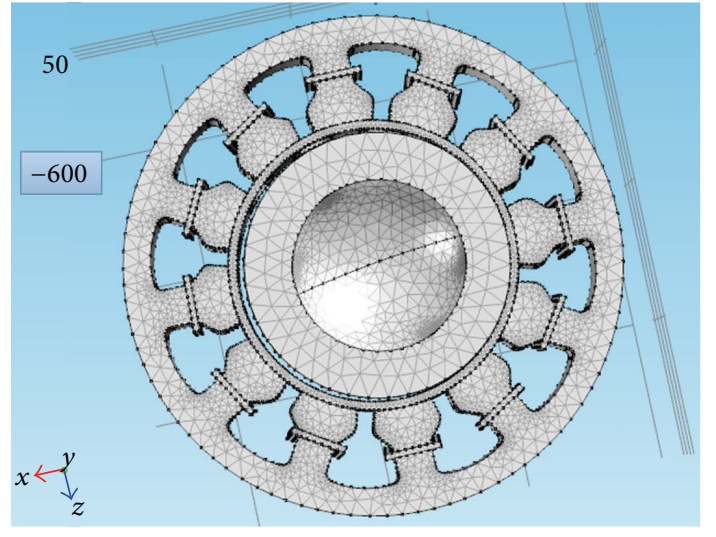

FIGURE 8: Grid of the dished rotor gyroscope.

Let the Gibbs energy of the pure aphasiac volume density be $g^{\alpha} \cdot g^{\beta}$ is the pure phase flow, $g^{I}$ is the interface phase, and $g^{\beta}-g^{\alpha}$ is the thermodynamics of the phase transformation driving force. This kind of GS for the volume density Gibbs energy system can be described as follows at any point in time [32]:

$$
g_{s}=w^{\alpha}(\phi) g^{\alpha}+w^{\beta}(\phi) g^{\beta}+w^{I}(\phi) g^{I} \text {. }
$$

Function relation is suitable to describe the thermodynamics of interface properties. They are $w^{\alpha}(\phi), w^{\beta}(\phi)$, and $w^{I}(\phi)$. The continuous function of weight has the following properties, which correspond to the total Gibbs energy as follows [32]:

$$
\begin{aligned}
& G_{S}=F \int_{V}(\operatorname{rad} \phi)^{2} d V \\
& \approx \frac{\Delta}{F} \sum_{x=2}^{X}(\phi(x)-\phi(x-1))^{2}, \\
& G=G_{S}+G_{\phi} .
\end{aligned}
$$

The partial derivative of $\phi(x), \phi(y)$, and $\phi(z)$ is determined as follows:

$$
\begin{aligned}
& \frac{\partial G}{\partial \phi(x)} \\
& =U(\phi(x)) \Delta\left(-24 \phi(x)+48 \phi^{2}(x)\right)\left(g^{\alpha}-g^{I}\right) \\
& +(1-U(\phi(x))) \Delta\left(24-72 \phi(x)+48 \phi^{2}(x)\right) \\
& \times\left(g^{I}-g^{\beta}\right)+\frac{2 F}{\Delta} \\
& \times(2 \phi(x)-\phi(x-1)-\phi(x+1)), \\
& \frac{\partial G}{\partial \phi(y)} \\
& =U(\phi(y)) \Delta\left(-24 \phi(y)+48 \phi^{2}(y)\right)\left(g^{\alpha}-g^{I}\right) \\
& +(1-U(\phi(y))) \Delta\left(24-72 \phi(y)+48 \phi^{2}(y)\right) \\
& \times\left(g^{I}-g^{\beta}\right)+\frac{2 F}{\Delta} \\
& \times(2 \phi(y)-\phi(y-1)-\phi(y+1)), \\
& \frac{\partial G}{\partial \phi(z)} \\
& =U(\phi(z)) \Delta\left(-24 \phi(z)+48 \phi^{2}(z)\right)\left(g^{\alpha}-g^{I}\right) \\
& +(1-U(\phi(z))) \Delta\left(24-72 \phi(z)+48 \phi^{2}(z)\right)
\end{aligned}
$$




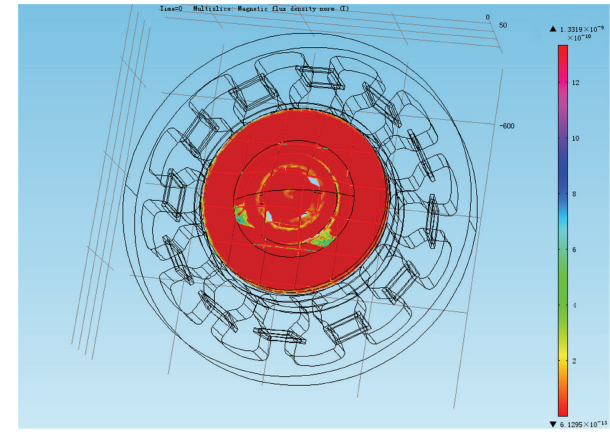

(a)

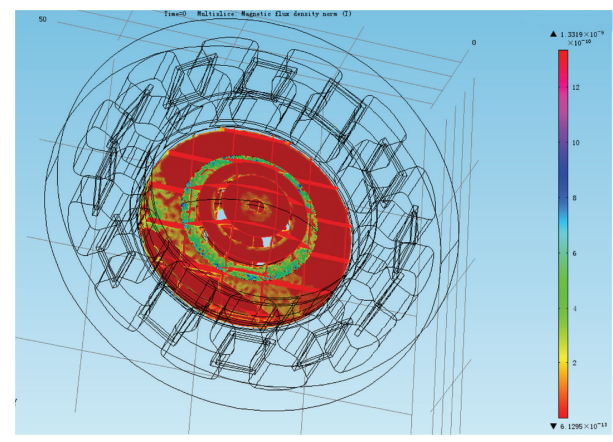

(b)

FIGURE 9: Thermal simulation of the dishing rotor gyroscope. (a) $5000 \mathrm{rad} / \mathrm{min}$ and (b) $5549 \mathrm{rad} / \mathrm{min}$.

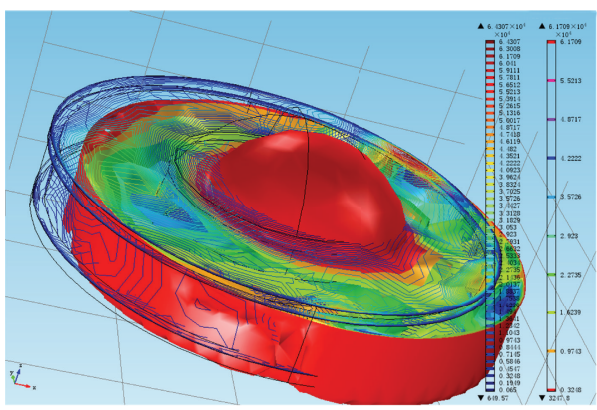

(a)

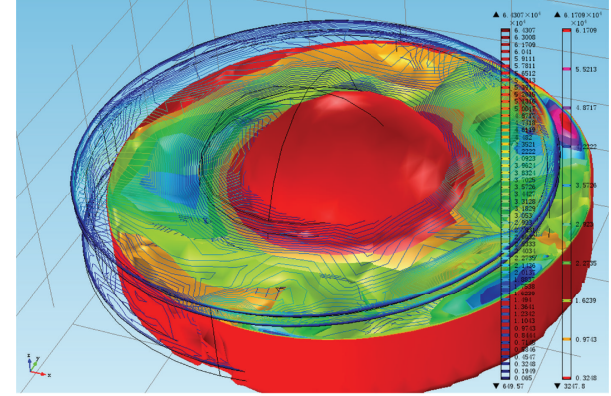

(b)

Figure 10: Bonding force simulation of the liquid floated gyroscope. (a) $5000 \mathrm{rad} / \mathrm{min}$ and (b) $5549 \mathrm{rad} / \mathrm{min}$.

$$
\begin{aligned}
& \times\left(g^{I}-g^{\beta}\right)+\frac{2 F}{\Delta} \\
& \times(2 \phi(z)-\phi(z-1)-\phi(z+1)) .
\end{aligned}
$$

For the heat dissipation system, the total dissipation is in the framework of linear nonequilibrium thermodynamics, as given above. Consider

$$
Q=A \int_{V} \dot{\phi}^{2} d V \approx A \Delta \sum_{x=1}^{X} \dot{\phi}^{2}(x)
$$

where

$$
\begin{gathered}
\dot{\phi}(x)=-\frac{1}{A \Delta} \frac{\partial G}{\partial \phi(x)}, \\
\gamma=G-\left(x_{\text {int }} g^{\alpha}+\left(X-x_{\text {int }}\right) g^{\beta}\right) .
\end{gathered}
$$

$\gamma$ is independent of energy, and $h$ is the thickness of the interface.

The value of the flow of $M$ is given by

$$
\begin{aligned}
Q & =\frac{v^{2}}{M}, \\
M & =\frac{v}{\left(g^{\beta}-g^{\alpha}\right)} .
\end{aligned}
$$

\section{Measurements of the Experiment}

5.1. Rough Hydrophobic Surface. The rotor's coupling heat is given in the theoretical calculation and the simulation above. The coupled heat is related to an experiment using PIV measurements, and the results are shown in Figures 14(a) and 14(b). The rotor operates at speeds of $5000 \mathrm{rad} / \mathrm{min}$ and $5549 \mathrm{rad} / \mathrm{min}$ and the rotor's flow field velocity distribution of heated.

Consider $R=2 \mathrm{~mm}$, as measured from the center, and the oil of the measurement experiment is for the rotor.

For the rotor operates at speeds of $5000 \mathrm{rad} / \mathrm{min}$ and $5549 \mathrm{rad} / \mathrm{min}$, and the rotor's flow field velocity distribution of heated. Now, it is marked for analysis, and the thermal maximum edge away from the speed of rotor is included $\left(88.13^{\circ}\right)$. The results are shown in Figures 15(a) and 15(b). Theoretical analysis and simulation of the thermal fields, it is that the rotor's maximum coupling thermal boundary, the maximum temperature of gyroscope rotor is that $88.13^{\circ} \mathrm{C}$.

5.2. Smooth Surface. Consider $R=2 \mathrm{~mm}$, as measured from the center. The measurements for the experiment of the rotor oil body are as follows. The rotor speed is $5549 \mathrm{rad} / \mathrm{min}$ given that it is located $2 \mathrm{~mm}$ away from the center. The results are shown in Figure 16.

The experimental results of the coupled thermal simulation and theoretical results are as follows. The maximum rotor coupled border coupling heat for the coupled thermal 


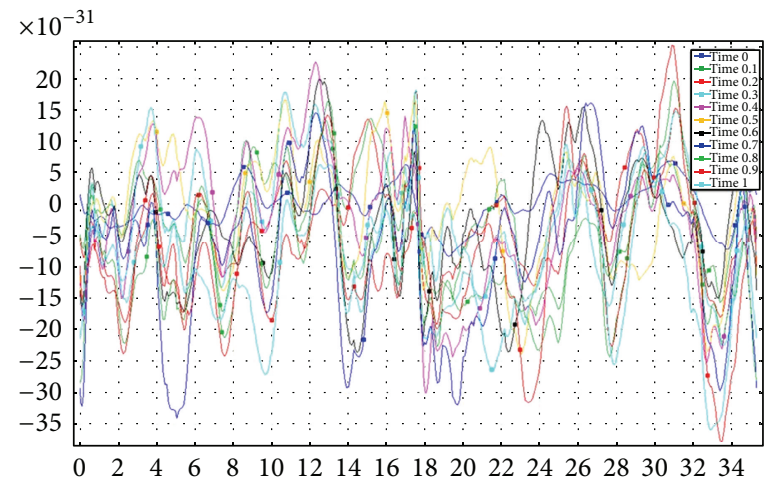

(a)

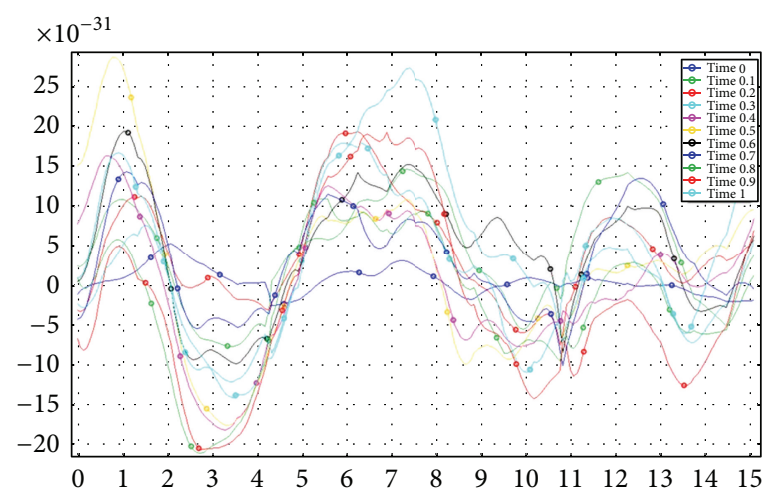

(b)

FIGURE 11: Bonding force distribution of the liquid floated gyroscope. (a) $5000 \mathrm{rad} / \mathrm{min}$ and (b) $5549 \mathrm{rad} / \mathrm{m}$.

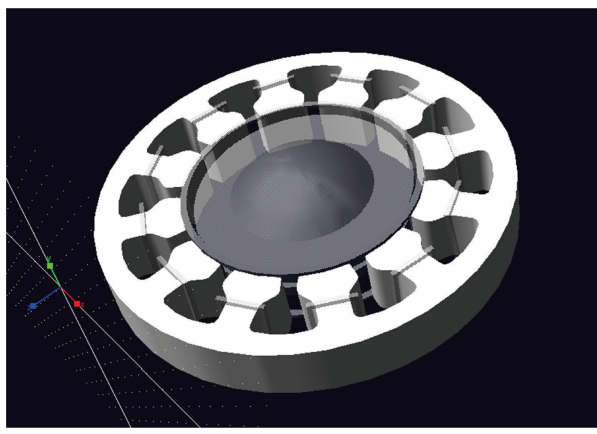

(a)

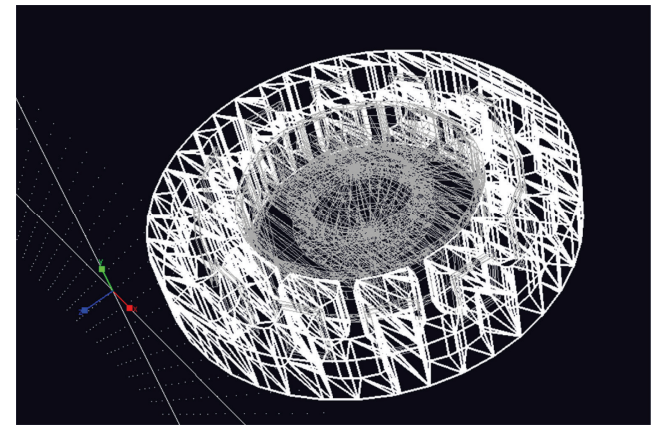

(b)

Figure 12: Grid of the liquid floated gyroscope.

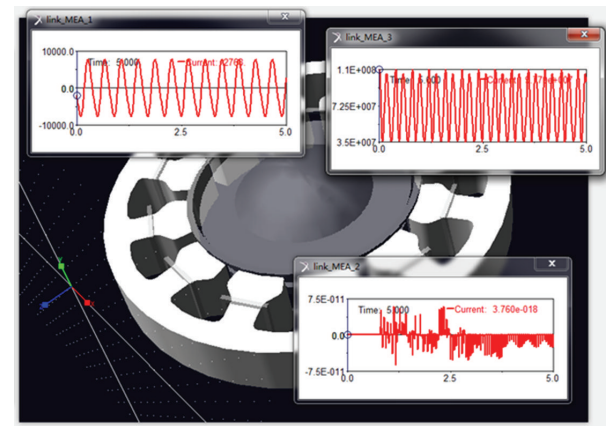

FIGURE 13: Simulation of the liquid floated gyroscope in ADAMS.

simulation results is $83.25^{\circ}$ (degree centigrade) and that for the rotor coupled heat results in the experiment is $88.13^{\circ}$ (degree centigrade). One of the biggest coupled thermal locations can be obtained by calculating the maximum stress, which is in $x, 1.4 ; y, 8.43$; $\min 97.23$; and $\max 154.34$. Two methods can be employed to improve the precision of the gyroscope, considering the rotor coupled thermal and dynamic characteristics. In the first method, the rotor boundary is processed into a $0.005 \mathrm{~mm}$ radian. In the second method, the rotor is processed with nanomaterials.

\section{Conclusions}

The dishing rotor gyroscope was coupled and was established in different coordinates of the system for the thermodynamic analysis. Dynamic equations were solved, and the dynamic equation of the disc rotor angular rate was obtained. The model theory was solved in the COMSOL through 2D and 3D simulations coupled with thermal simulation, and it was in the mechanics of the dynamic system simulation in the ADAMS components. Maximum stress was found in $x, 1.4 ; y, 8.43$; $\min 97.23$; and $\max 154.34$. The maximum stress occurred in the dishing rotor border at a high-speed rotation. The secondary flow reached $5549 \mathrm{r} / \mathrm{min}$, and the heat generated increased. Meanwhile, the high-speed rotation of the rotor was volatile, and the dishing rotor movement was unstable. Adding nonmaterial processed at high temperature at the top and the bottom surfaces of the dished rotor is required.

\section{Conflict of Interests}

The authors declare that there is no conflict of interests regarding the publication of this paper. 


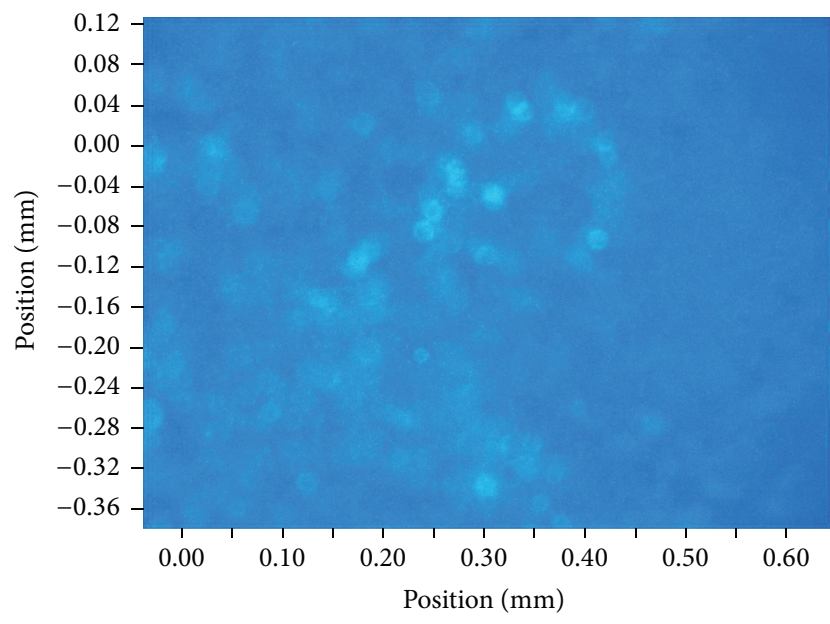

(a)

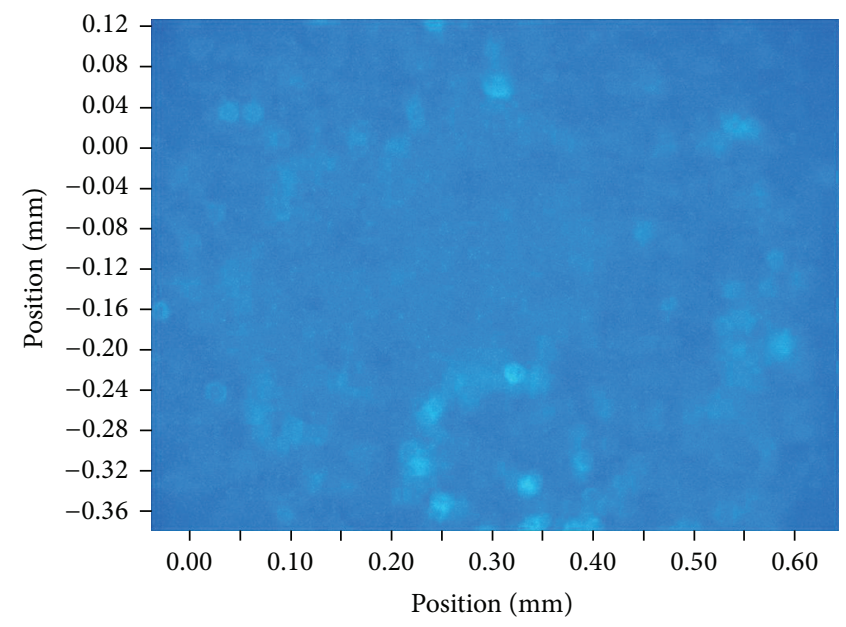

(b)

FIGURE 14: Thermal fluid measurement of the dished rotor. (a) $5000 \mathrm{rad} / \mathrm{min}$ and (b) $5549 \mathrm{rad} / \mathrm{min}$.

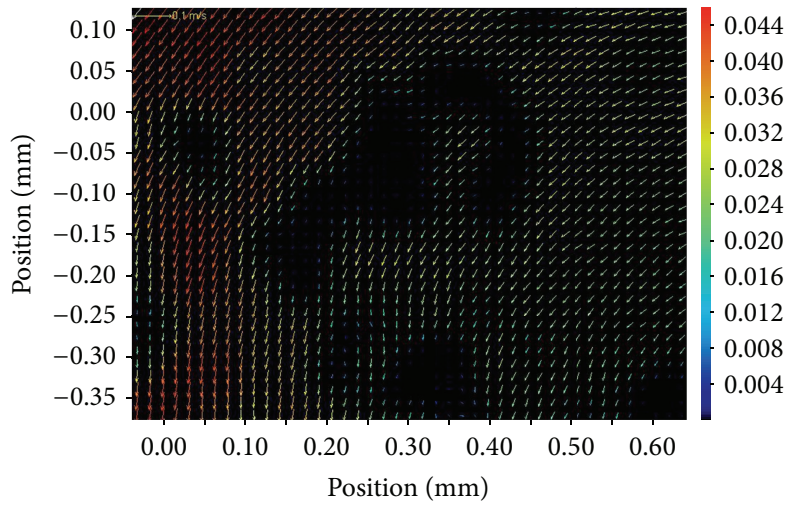

(a)

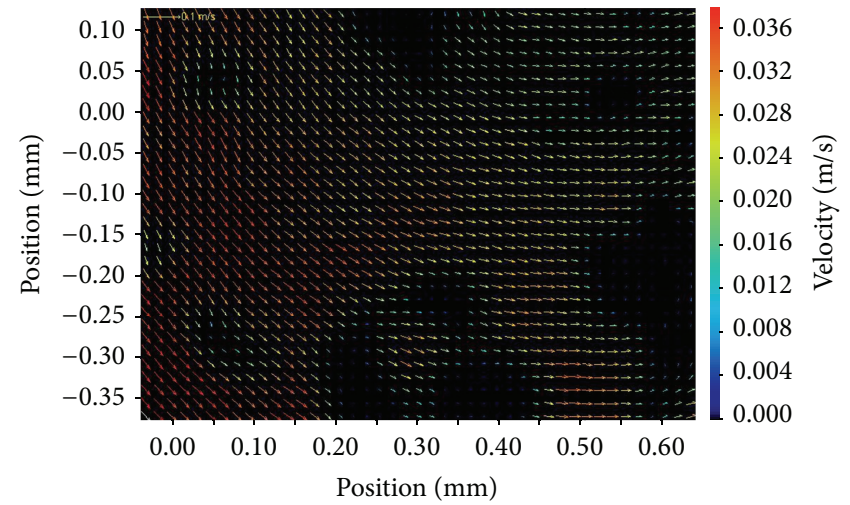

(b)

FIGURE 15: Thermodynamic analysis of the measuring fluid for the dishing rotor. (a) $5000 \mathrm{rad} / \mathrm{min}$ and (b) $5549 \mathrm{rad} / \mathrm{min}$.

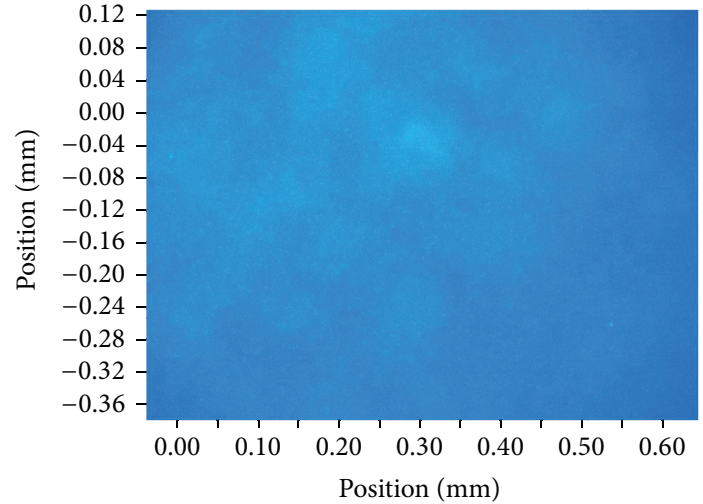

(a)

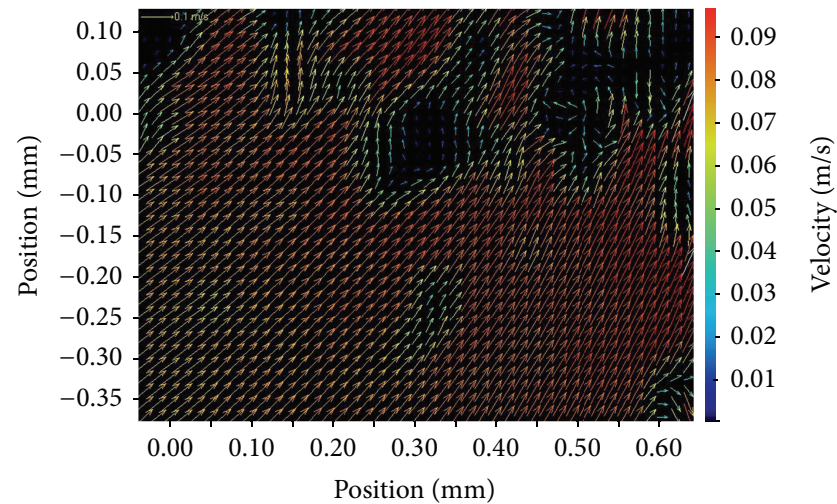

(b)

Figure 16: Thermal fluid experimental results of the dishing rotor measurements (a) $5549 \mathrm{rad} / \mathrm{min}$ and (b) thermal fluid experimental of rotor in $5549 \mathrm{rad} / \mathrm{min}$. 


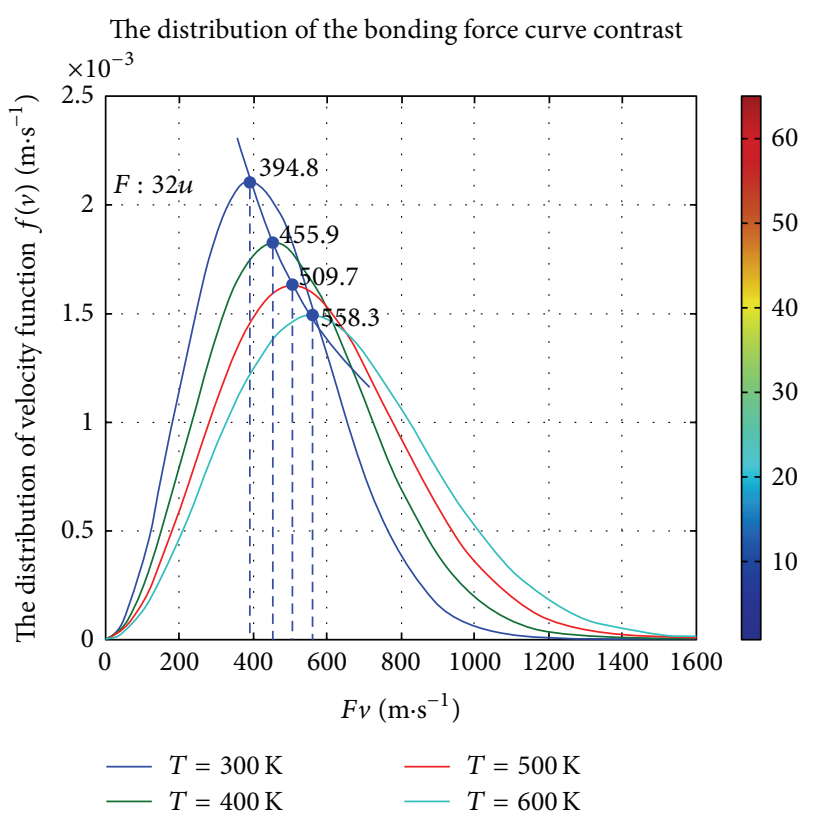

FIGURE 17: Stress analysis of the rotor in the experiment with the bonding thermal force of rotor in $5549 \mathrm{rad} / \mathrm{min}$.

\section{Acknowledgment}

This work was supported by the Ministry of Science and Technology of China (Grant nos. 2012CB934103 and 2011ZX02403).

\section{References}

[1] D. Xia, C. Yu, and L. Kong, "The development of micromachined gyroscope structure and circuitry technology," Sensors, vol. 14, no. 1, pp. 1394-1473, 2014.

[2] N. Yezdi, F. Anasazi, and K. Naafi, "Micro-machined inertial sensors," Proceedings of the IEEE, vol. 86, no. 8, pp. 1640-1659, 1998.

[3] K. Liu, W. Zhang, W. Chen et al., "The development of micro-gyroscope technology," Journal of Micromechanics and Microengineering, vol. 19, no. 11, Article ID 113001, 2009.

[4] L. Yan, Z. Xiao, F. Zhang et al., "Advances of silicon-based integrated photonic devices and applications in optical gyroscope and optical communication," Chinese Journal of Lasers, vol. 36, no. 3, pp. 547-553, 2009.

[5] A. Sharma, M. F. Zaman, M. Zucher, and F. Ayazi, "A 0.1०/HR bias drift electronically matched tuning fork microgyroscope," in Proceedings of the 21st IEEE International Conference on Micro Electro Mechanical Systems (MEMS '08), pp. 6-9, Tucson, AZ, USA, January 2008.

[6] L. Xie, D. Xiao, and H. Wang, "Sensitivity analysis and structure design for tri-mass structure micromachined gyroscope," in Proceedings of the 4th IEEE International Conference on Nano/Micro Engineered and Molecular Systems, vol. 5, pp. 126129, Shenzhen, China, 2009.

[7] F. L. Che, B. Xiong, Y. F. Li, and Y. L. Wang, "A novel electrostatic-driven tuning fork micromachined gyroscope with a bar structure operating at atmospheric pressure," Journal of Micromechanics and Microengineering, vol. 20, no. 1, pp. 1-6, 2010.

[8] Z. Y. Guo, Z. C. Yang, Q. C. Zhao et al., "A lateral-axis micromachined tuning fork gyroscope with torsional Z-sensing and electrostatic force-balanced driving," Journal of Micromechanics and Microengineering, vol. 20, no. 2, Article ID 025007, 2010.

[9] Z. Y. Guo, Z. C. Yang, Q. C. Zhao et al., "A lateral-axis micromachined tuning fork gyroscope with torsional Z-sensing and electrostatic force-balanced driving," Journal of Micromechanics and Microengineering, vol. 20, no. 2, Article ID 025007, pp. 1-7, 2010.

[10] F. Ayazi and K. Najafi, "Design and fabrication of highperformance polysilicon vibrating ring gyroscope," in Proceedings of the 11th IEEE Annual International Workshop on Micro Electro Mechanical Systems (MEMS '98), pp. 621-626, Heidelberg, Germany, January 1998.

[11] F. Ayazi and K. Najafi, "A HARPSS polysilicon vibrating ring gyroscope," Journal of Microelectromechanical Systems, vol. 10, no. 2, pp. 169-179, 2001.

[12] G. He and K. Najafi, "A single-crystal silicon vibrating ring gyroscope," in Proceedings of the 15th IEEE International Conference on Micro Electro Mechanical Systems (MEMS '02), pp. 718-721, Las Vegas, Nev USA, January 2002.

[13] D. Piyabongkarn, R. Rajamani, and M. Greminger, "The development of a MEMS gyroscope for absolute angle measurement," IEEE Transactions on Control Systems Technology, vol. 13, no. 2, pp. 185-195, 2005.

[14] I. P. Prikhodko, S. A. Zotov, A. A. Trusov, and A. M. Shkel, "Foucault pendulum on a chip: rate integrating silicon MEMS gyroscope," Sensors and Actuators A: Physical, vol. 177, pp. 6778, 2012.

[15] X. Wu, W. Chen, Y. Lu et al., "Vibration analysis of a piezoelectric micromachined modal gyroscope (PMMG)," Journal of Micromechanics and Microengineering, vol. 19, Article ID 125008, pp. 1-10, 2009.

[16] T. Murakoshi, Y. Endo, K. Fukatsu, and S. Nakamura, "Electrostatically levitated ring-shaped rotational-gyro/accelerometer," Japanese Journal of Applied Physics, vol. 42, no. 4, pp. 2468-2472, 2003.

[17] F. T. Han, Y. F. Liu, L. Wang, and G. Y. Ma, "Micromachined electrostatically suspended gyroscope with a spinning ringshaped rotor," Journal of Micromechanics and Microengineering, vol. 22, no. 10, Article ID 105032, pp. 1-9, 2012.

[18] G. Xue, X. Zhang, and H. Zhang, "Electromagnetic design of a magnetically suspended gyroscope prototype," in Proceedings of the International Conference on Applied Superconductivity and Electromagnetic Devices (ASEMD '09), pp. 369-372, Chengdu, China, September 2009.

[19] A. N. Gorban and I. V. Karlin, "Method of invariant manifold for chemical kinetics," Chemical Engineering Science, vol. 58, no. 21, pp. 4751-4768, 2003.

[20] D. V. Tunitsky, "Reducing quasilinear systems to block triangular form," Sbornik: Mathematics, vol. 204, pp. 438-462, 2013.

[21] L. Ardizzone, G. Gaeta, and M. S. Mongiovì, "A continuum theory of superfluid turbulence based on extended thermodynamics," Journal of Non-Equilibrium Thermodynamics, vol. 34, no. 3, pp. 277-297, 2009.

[22] V. Triani and V. A. Cimmelli, "Entropy principle, non-regular processes, and generalized exploitation procedures," Journal of Mathematical Physics, vol. 53, no. 6, Article ID 063509, 2012. 
[23] C.-H. Liu, K.-H. Lin, H.-C. Mai, and C.-A. Lin, "Thermal boundary conditions for thermal lattice Boltzmann simulations," Computers and Mathematics with Applications, vol. 59, no. 7, pp. 2178-2193, 2010.

[24] R. Mei, W. Shyy, D. Yu, and L.-S. Luo, "Lattice Boltzmann Method for 3-D Flows with Curved Boundary," Journal of Computational Physics, vol. 161, no. 2, pp. 680-699, 2000.

[25] G. R. McNamara, A. L. Garcia, and B. J. Alder, "Stabilization of thermal lattice Boltzmann models," Journal of Statistical Physics, vol. 81, no. 1-2, pp. 395-408, 1995.

[26] X. He, S. Chen, and G. D. Doolen, "A novel thermal model for the lattice Boltzmann method in incompressible limit," Journal of Computational Physics, vol. 146, no. 1, pp. 282-300, 1998.

[27] P. Lallemand and L.-S. Lou, "Hybrid finite-difference thermal lattice Boltzmann equation," International Journal of Modern Physics B, vol. 17, no. 1-2, pp. 41-47, 2003.

[28] P. Lallemand and L.-S. Luo, "Theory of the lattice Boltzmann method: acoustic and thermal properties in two and three dimensions," Physical Review E: Statistical, Nonlinear, and Soft Matter Physics, vol. 68, no. 3, pp. 706-709, 2003.

[29] G. R. McNamara, A. L. Garcia, and B. J. Alder, "Stabilization of thermal lattice Boltzmann models," Journal of Statistical Physics, vol. 81, no. 1-2, pp. 395-408, 1995.

[30] E. Fermi, Thermodynamics, Dover, New York, NY, USA, 1956.

[31] S. Abe, "Heat and entropy in nonextensive thermodynamics: transmutation from Tsallis theory to Rényi-entropy-based theory," Physica A: Statistical Mechanics and Its Applications, vol. 300, no. 3-4, pp. 417-423, 2001.

[32] J. Svoboda, F. D. Fischer, and D. L. McDowell, "Derivation of the phase field equations from the thermodynamic extremal principle," Acta Materialia, vol. 60, no. 1, pp. 396-406, 2012. 


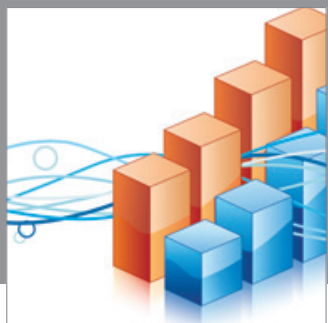

Advances in

Operations Research

mansans

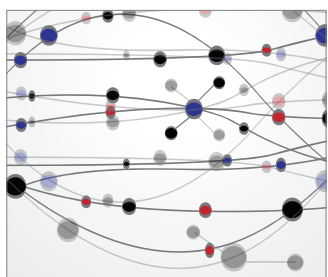

The Scientific World Journal
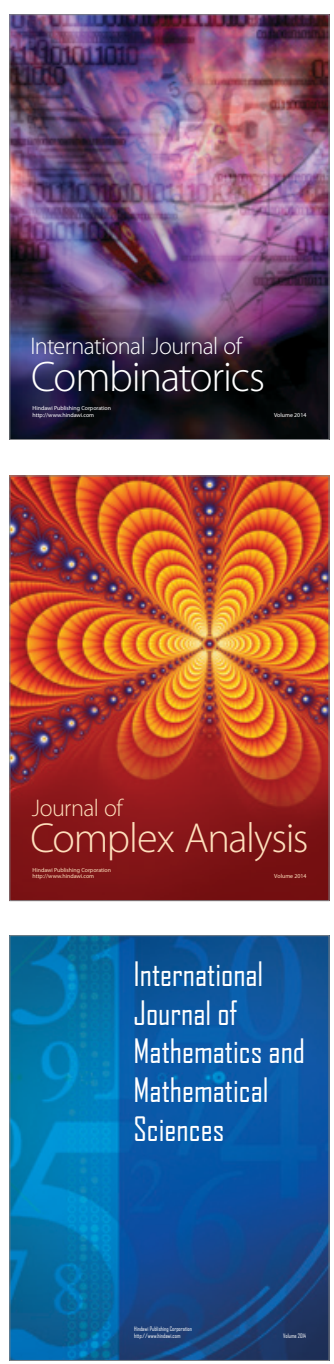
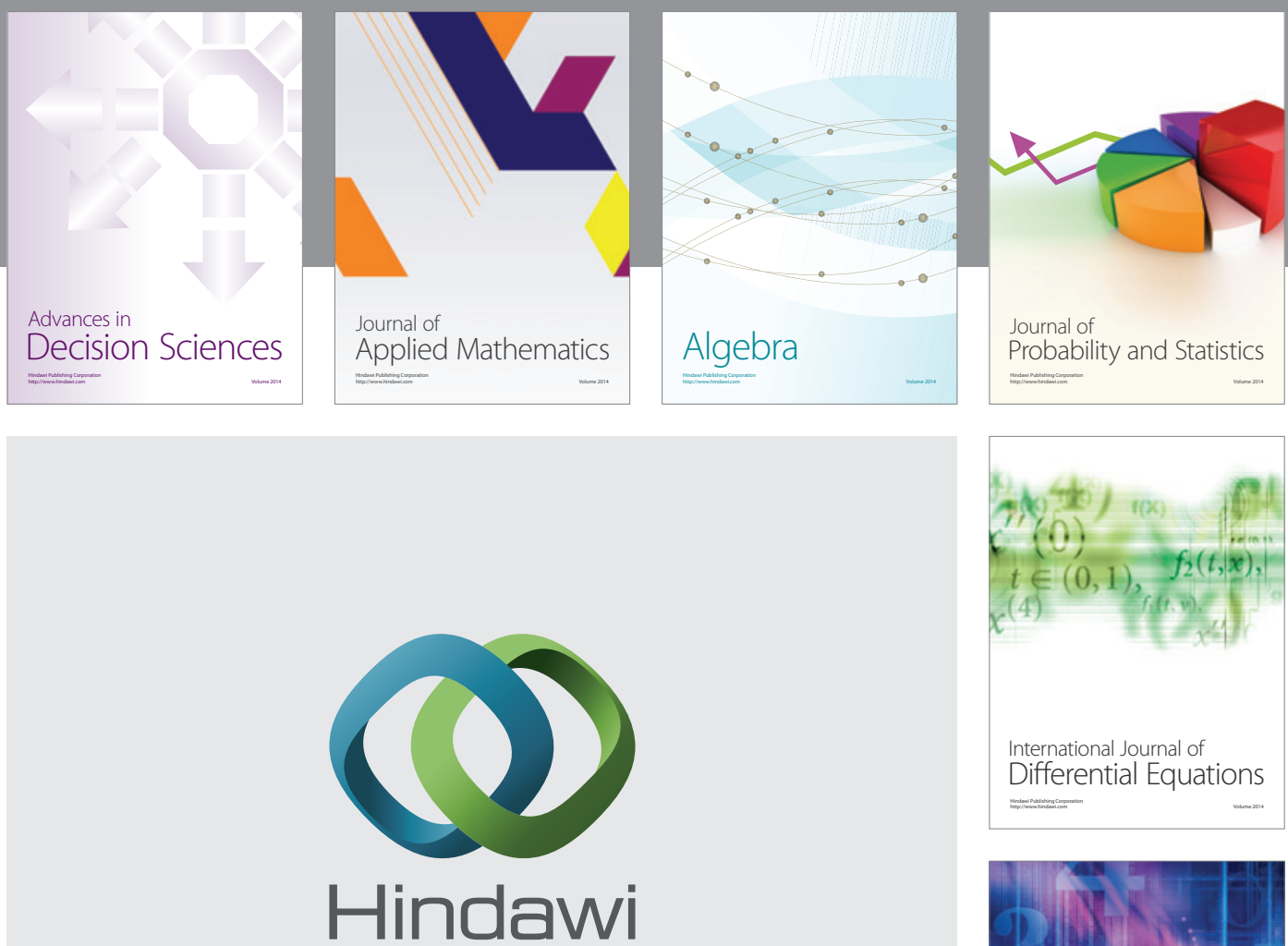

Submit your manuscripts at http://www.hindawi.com
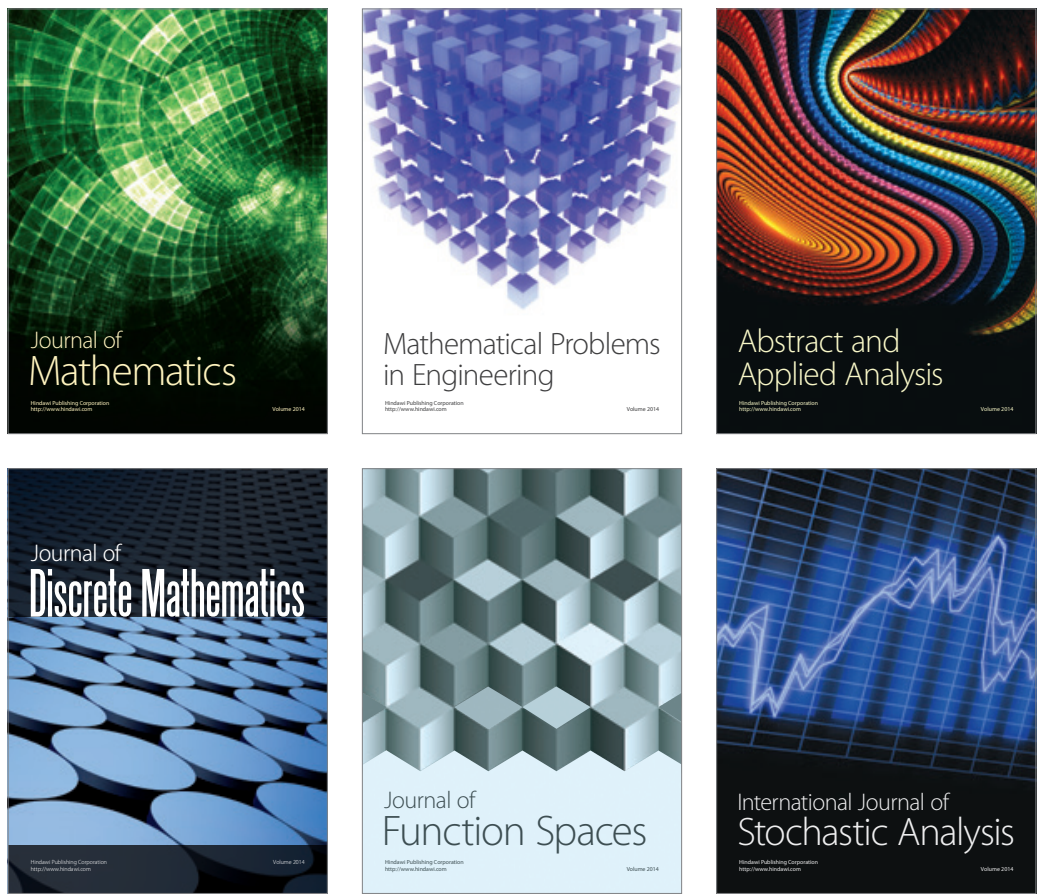

Journal of

Function Spaces

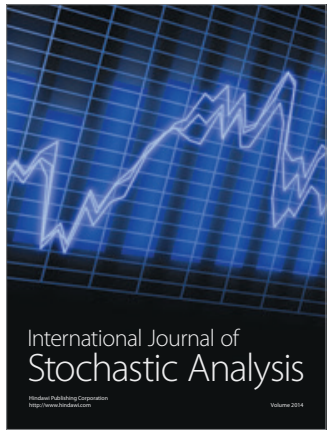

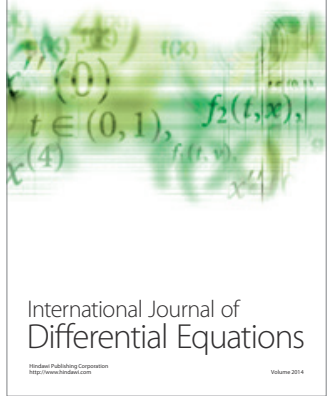
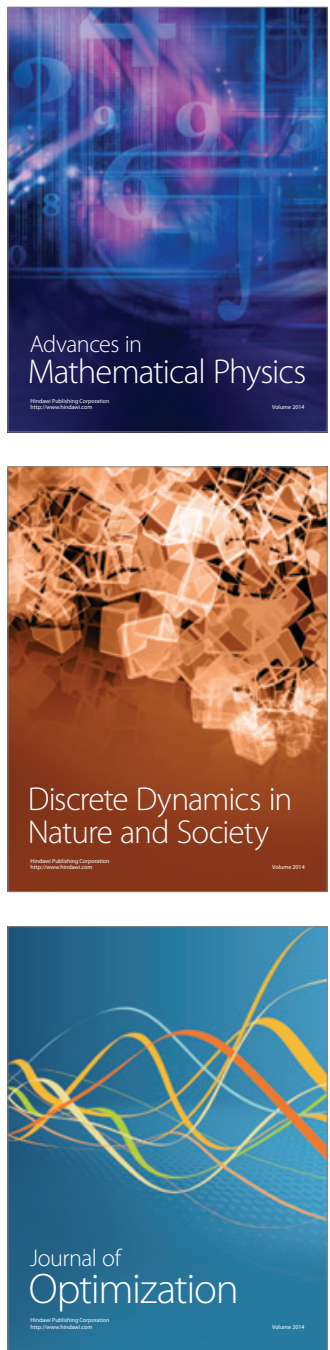